The Behavioural Components of Risk Aversion

Greg B. Davies and Stephen E. Satchell

October 2004

CWPE 0458

Not to be quoted without permission 


\title{
The Behavioural Components of Risk Aversion
}

\author{
Greg B Davies* \\ Stephen E Satchell \\ Faculty of Economics and Politics Faculty of Economics and Politics \\ University of Cambridge \\ University of Cambridge
}

July 12, 2004

\begin{abstract}
Corresponding Author: Greg B Davies, Trinity Hall, Cambridge, CB2 1TJ, UK

Tel: $+44(0) 7881827355$

e-mail: gbd21@cam.ac.uk

* The first author would like to thank the ESRC for funding.
\end{abstract}




\begin{abstract}
The risk premium is affected by loss aversion and probability distortions as well as utility curvature. We introduce two variants - the total risk premium relative to objective expected value, and the subjective risk premium relative to perceived expected value. Approximate solutions for each provide analogies to the Pratt-Arrow coefficient of risk aversion (showing how risk attitude depends on each behavioural component), and sufficient conditions for risk aversion. Earlier results of Levy and Levy (2002) which examined decision weights in isolation are revised and extended to show how the curvature and loss aversion conditions are affected by probability distortions.
\end{abstract}

Keywords: Risk-Premium; Cumulative Prospect Theory; Loss Aversion; Decision Weights; Utility Curvature

JEL Subject Categories: D81 
It is a commonly held belief amongst economists that humans are, or should be risk averse. Simply put this usually means that, given the choice between a certain amount of money and a gamble for which the expectation is the same amount of money, a risk averse individual will choose certainty. For most people, introspection reveals this to be quite plausible in many situations, although risk-seeking behaviour has often been observed in experimental studies of human choice. In standard economic models the acceptability of risk aversion has been increased by the fact that it is a necessary conclusion if people face diminishing marginal utility, an assumption for which the intuitive appeal is much stronger. It was seldom questioned, however, whether it is reasonable to associate human risk attitude entirely with the propensity to appreciate each unit of consumption by slightly less as the total amount increases. Surely an attitude to risk involves somewhat more than diminishing marginal returns? Recent developments have introduced more sophisticated and empirically accurate models of human choice which break this strict one-toone link and enable us to examine more closely the question of what underlying behavioural factors determine risk attitude. These factors still include diminishing sensitivity to amounts of money or consumption, but add to this the human tendency to be more concerned with losses than with gains, as well as psychological effects such as hope and fear, which draw our attention respectively towards extremely good, and extremely bad outcomes in a gamble.

The notion of the risk premium of a gamble has provided a useful way of characterising the degree of risk aversion of an individual facing a risky choice. It is traditionally defined as the amount by which the expected value of the gamble exceeds the certainty equivalent value. Thus, the risk premium is the certain amount that an individual would give up in order to avoid the risk inherent in the gamble. A positive risk premium may be taken to be indicative of risk aversion. In the standard framework of Expected Utility Theory (EUT), the risk premium has a particularly simple interpretation. K. Arrow (1965) and J. Pratt (1964) showed that, approximately, the risk premium depends only on the variance of the lottery, and on the "coefficient of absolute risk aversion" which reflects the shape of the decision maker's utility function. More fundamentally, the risk premium is positive if and only if the utility function is concave. Thus, in continuous form EUT risk averse or risk seeing behaviour is governed entirely by the concavity or convexity respectively of the (increasing) utility function.

In recent decades, the simple framework of EUT has been found to provide a poor description 
of actual behaviour in risky situations and has undergone a number of revisions that attempt to remedy this descriptive failure. Amongst these remedies (which have not yet been completely successful in the quest for descriptive adequacy (see Davies 2004)) have been the introduction of utility seen in terms of changes from a reference point rather than in terms of absolute wealth levels (Kahneman and Tversky 1979), and the related notion of Loss Aversion. These changes have reflected greater psychological sophistication in the way in which utilities are deemed to be attached to monetary outcomes. A further significant shift was the recognition that individuals may distort objective or subjective probabilities when making decisions due to their attention being disproportionately directed to the outcomes in the tails of the distribution of outcomes. This resulted in the suite of Rank-Dependent Utility (RDU) models of which the first was described by Quiggin (1982).

These new elements introduced into EUT have the result that risk aversion can no longer be simply described in terms of the curvature of the utility function. Both loss aversion and non-linear probability distortions add new sources of risk attitude to that traditionally derived from the concavity of the utility function. In addition, the utility function itself may display quite different characteristics for losses than for gains. Thus, whilst the sign of the risk premium may still be taken to reflect the overall risk attitude (either risk aversion or risk seeking), this sign is determined by a complex interaction of a number of independent sources of risk aversion.

This paper examines the extension of the concepts of risk aversion within the framework of Cumulative Prospect Theory (CPT) (Tversky and Kahneman 1992) which combines reference dependence, loss aversion and rank-dependence. The effect of the probability weighting component of CPT on the risk premium has already been partially investigated by Levy and Levy (2002), although their application of CPT is flawed in places. This paper will both correct and extend their analysis. In continuous CPT (Davies and Satchell 2002) the question of whether the subject is risk averse or risk seeking for a given prospect may depend in a complex way on the interaction between the utility functions for gains and losses, the probability weighting function, and the shape of the distribution.

In section 1 below we introduce continuous CPT and its behavioural components of reference dependence, loss aversion, utility curvature and subjective probability distortions. We then define and provide general solutions for both the objective risk premium which reveals the true 
amount of risk attitude displayed by the decision maker, and the subjective risk premium which indicates the decision maker's own assessment of her risk attitude. The difference between these two concepts we term the subjectivity premium.

In section 2 we follow Pratt's (1964) methodology for obtaining approximate local solutions for the risk premium. We analyse first in section 2.1 the situation in which there is no reweighting of probabilities, and provide the sufficient conditions on loss aversion and the shape of the utility functions to guarantee local risk aversion at the reference point. These conditions are analogous to the traditional Pratt-Arrow condition of concavity of the utility function, but in our more complex behavioural model restrictions are required on both loss aversion, and the curvature of gains and losses separately. The Pratt-Arrow risk premium is shown to be a special case of the CPT risk premium. For completeness we provide a characterisation for local risk aversion away from the reference point, and show that this collapses to the special case of the standard Pratt-Arrow risk premium.

Thereafter in section 2.2 we introduce the full CPT model with decision weights and discuss the previous work of Levy and Levy in this regard before providing comprehensive approximate results for both the objective and subjective risk premia at the reference point. The introduction of probability distortions changes the sufficiency conditions that apply without decision weights such that they are reliant on the way in which the probability distribution is distorted, and therefore on the slope of the decision weighting function at the reference point. We show how the conditions are altered by the asymmetry (through the probability of obtaining a gain rather than a loss) of the underlying distributions.

Section 3 provides an example illustrating the role of the components of the risk premium in financial decision making where specific assumptions are made regarding the parametric forms of the utility and probability weighting functions. Section 4 concludes. 


\section{Behavioural Risk Aversion}

\subsection{Cumulative Prospect Theory}

Under continuous cumulative prospect theory a continuous prospect $\Psi$ with possible outcomes distributed on the set of real numbers is evaluated in relation to a reference point using a utility function that is defined over the change that each possible outcome represents from the reference point, and a non-linear transformation of the probability distribution. In this formulation a single prospect will be evaluated differently from different reference points. This dependence on the reference point has been frequently referred to in the literature, but little work has attempted for formalise the relationship between the reference point and the evaluation of prospects (recent exceptions being Schmidt 2003 and Sugden 2003). The valuation of prospect $\Psi$ in continuous Cumulative Prospect Theory (CPT) may be given by:

$$
V_{y}[\Psi]=\int v(x) m(x) f(x) d x
$$

where the outcomes values, $x$, are the deviations of the possible values of the distribution (represented by $\Psi$ ) from the reference point. Thus, if the reference point is current wealth $y=W_{0}$ then the values taken by the random variable $x$ will be the absolute outcomes of $\Psi$ with negative outcomes coded as losses from the status quo point and positive outcomes coded as gains. If, as is a more frequent assumption in finance, the reference point is the future value of current wealth if invested at the risk free rate $r_{f}$ until the resolution of the prospect (to account for the value of time), then $x$ are the deviations from $y=W_{0}\left(1+r_{f}\right)$ where $r_{f}$ is the risk free rate that applies the period of time between the decision point, and the resolution of the prospect.

$v(x)$ is the value to the decision maker of each possible realised outcome $x$.

$$
v(x)=\left\{\begin{array}{c}
v_{+}(x), \text { if } x>0 \\
0, \text { if } x=0 \\
\lambda v_{-}(x), \text { if } x<0
\end{array}\right.
$$

It is continuous and monotonically increasing with $v(0)=0$. The most common restrictions on the form of $v(x)$ are diminishing sensitivity away from the reference point (i.e., it is concave 
for gains and convex for losses) and loss aversion (it is steeper for losses than for gains). These distinctions between losses and gains require separate functions to be used above and below the reference point. Following Köbberling and Wakker (2003) we separate out the risk attitude that arises from the attribution of basic utility to gains and losses (achieved through $v_{+}(x)$ and $v_{-}(x)$ ) from that due to loss aversion (governed by $\lambda$ ). With this decomposition it is possible to have two individuals with identical risk attitudes to all gains-only and loss-only prospects, but different attitudes with regard to mixed prospects. Loss aversion exists for $\lambda>1$ and $V_{y}[\Psi ; \lambda]$ is decreasing in $\lambda$ as $v_{-}(x)<0$ for all $x<0$.

$m(x)$ is a multiplier of the objective probability density function, $f(x)$, that reflects rankdependent distortions of objective probability to result in an imputed subjective distribution (see Davies and Satchell 2003). It is defined as follows:

$$
m(x)=\left\{\begin{array}{c}
m_{+}(x)=w_{+}^{\prime}(1-F(x)) \text { for all } x \geq 0 \\
m_{-}(x)=w_{-}^{\prime}(F(x)) \text { for all } x<0
\end{array}\right.
$$

$w_{+}$and $w_{-}$are two strictly increasing weighting functions $[0,1] \times \mathbb{R} \rightarrow[0,1]$ with $w_{+}(0)=$ $w_{-}(0)=0$ and $w_{+}(1)=w_{-}(1)=1$. They govern the way in which the cumulative probabilities are transformed subjectively and in Tversky and Kahneman's original model are presumed to be inverse-S shaped which is indicative of increased attention being given to the extreme outcomes of the distribution. $F(x)$ is the cumulative probability distribution of $x$, and $f(x)$ the associated density function.

\subsection{Risk Aversion}

Risk aversion is assessed according to whether the subject's evaluation of the prospect $\Psi$ is greater than or less than the utility of the expected value of the prospect. Thus, risk aversion may be characterised as:

$$
\begin{aligned}
v\left(E_{y}[\Psi]\right)> & V_{y}[\Psi], \text { or, } \\
v\left(\int x f(x) d x\right)> & \int_{-\infty}^{0} \lambda v_{-}(x) m_{-}(x) f(x) d x \\
& +\int_{0}^{\infty} v_{+}(x) m_{+}(x) f(x) d x
\end{aligned}
$$


The left hand side is the evaluation of the mathematical expected value of the distribution. This evaluation uses the same value function, $v$, as on the right hand side and, as such, the shape differs according to whether the expected value is positive or negative. Notice, too, that the expectation is taken for the outcomes, $x$, defined as the deviation of the prospect outcomes from the reference point. Thus $E_{y}[\Psi]$ is dependent on the reference point. The right hand side is the subjects evaluation of the prospect according to CPT. If we make a further assumption that probability is always treated objectively, then this model may be considerably simplified as $m_{-}$and $m_{+}$are everywhere equal to 1.

Using this formulation the risk premium $r^{\Psi}$, for the prospect $\Psi$ is the certain amount the decision maker would require in order to be indifferent between choosing the prospect $\Psi$, or receiving an amount equal to its expected value minus the risk premium. If $r^{\Psi}$ is positive then the decision maker is said to be risk averse.

$r^{\Psi}$ for $\Psi$ is defined by:

$$
\begin{aligned}
v\left(E_{y}[\Psi]-r^{\Psi}\right)= & V_{y}[\Psi], \text { or, } \\
v\left(\int x f(x) d x-r^{\Psi}\right)= & \int_{-\infty}^{0} \lambda v_{-}(x) m_{-}(x) f(x) d x \\
& +\int_{0}^{\infty} v_{+}(x) m_{+}(x) f(x) d x
\end{aligned}
$$

Adapting a concept introduced by Hilton (1988) we also define the subjective risk premium, $r_{S}^{\Psi}$ in this context:

$$
v\left(E_{y}^{s}[\Psi]-r_{S}^{\Psi}\right)=V_{y}[\Psi]
$$

$E_{y}^{s}[\Psi]$ is the subjective expected value of the prospect by the decision maker:

$$
E_{y}^{s}[\Psi]=\int x m(x) f(x) d x
$$

rather than the objective expected utility $E_{y}[\Psi]=\int x f(x) d x$.

This allows us to separate out the effect of subjective probability weighting so that $r_{S}^{\Psi}$ rep- 
resents the traditional Pratt-Arrow risk premium for that decision maker. That is, the risk premium when the decision maker's subjective probability assessment is taken to be the point of comparison. The sign of this Pratt-Arrow risk premium will be different depending on whether the decision maker believes herself to be risk averse or risk seeking. This may differ from whether the decision maker is actually risk seeking or risk averse in an objective sense, relative to the true expected value. It is the subjective risk premium that is employed by Levy and Levy (2002). We shall investigate both variations below.

\subsubsection{General Solutions}

Thus far we have characterised the risk attitudes of the decision maker with respect to the expected value of the prospect which is faced. In other words it tells us whether the decision maker would prefer the expected value of the prospect to the prospect itself and by how much.

We can use the definition of the risk premium (5) to examine the concept further in the case of reference dependent preferences. The objective risk premium is given by:

$$
r_{S}^{\Psi}=E_{y}[\Psi]-v^{-1}\left(V_{y}[\Psi]\right)
$$

and risk aversion in general implies

$$
\begin{aligned}
E_{y}[\Psi]-v^{-1}\left(V_{y}[\Psi]\right) & >0 \\
v\left(E_{y}[\Psi]\right) & >V_{y}[\Psi]
\end{aligned}
$$

To examine these concepts further we note that there are four possible cases.

Case 1a: $r^{\Psi}<E_{y}[\Psi]$ and $E_{y}[\Psi]>0$. Note that in this case the expected value of the prospect is necessarily positive if the decision maker is risk averse (as $r^{\Psi}>0$ ) and that the subjective evaluation of the prospect, $V_{y}[\Psi]$, is positive. The left hand side of (5) is evaluated using the gains value function: 


$$
\begin{aligned}
v_{+}\left(E_{y}[\Psi]-r^{\Psi}\right) & =V_{y}[\Psi] \\
r^{\Psi} & =E_{y}[\Psi]-v_{+}^{-1}\left(V_{y}[\Psi]\right)
\end{aligned}
$$

In this case risk aversion implies:

$$
\begin{aligned}
E_{y}[\Psi]-v_{+}^{-1}\left[V_{y}[\Psi]\right] & >0 \\
v_{+}\left(E_{y}[\Psi]\right) & >V_{y}[\Psi]
\end{aligned}
$$

and risk seeking $\left(r^{\Psi}<0<E_{y}[\Psi]\right)$ implies

$$
v_{+}\left(E_{y}[\Psi]\right)<V_{y}[\Psi]
$$

Case 1b: If $r^{\Psi}<E_{y}[\Psi]$ and $E_{y}[\Psi]<0$ the decision maker is necessarily risk seeking. In this case

$$
r^{\Psi}=E_{y}[\Psi]-v_{+}^{-1}\left(V_{y}[\Psi]\right)
$$

and risk seeking has

$$
\lambda v_{-}\left(E_{y}[\Psi]\right)>V_{y}[\Psi]
$$

Case 2a: If $r^{\Psi}>E_{y}[\Psi]$ and $E_{y}[\Psi]<0$ we are in the domain of losses and the evaluation becomes:

$$
\begin{aligned}
\lambda v_{-}\left(E_{y}[\Psi]-r^{\Psi}\right) & =V_{y}[\Psi] \\
r^{\Psi} & =E_{y}[\Psi]-v_{-}^{-1}\left(\frac{V_{y}[\Psi]}{\lambda}\right)
\end{aligned}
$$

risk aversion implies: 


$$
\begin{aligned}
E_{y}[\Psi]-v_{-}^{-1}\left(\frac{V_{y}[\Psi]}{\lambda}\right) & >0 \\
\lambda v_{-}\left(E_{y}[\Psi]\right) & >V_{y}[\Psi]
\end{aligned}
$$

and for risk seeking behaviour:

$$
\lambda v_{-}\left(E_{y}[\Psi]\right)<V_{y}[\Psi]
$$

Case 2b: Finally, if $r^{\Psi}>E_{y}[\Psi]$ and $E_{y}[\Psi]>0$ only risk aversion is possible:

$$
r^{\Psi}=E_{y}[\Psi]-v_{-}^{-1}\left(\frac{V_{y}[\Psi]}{\lambda}\right)
$$

and

$$
v_{+}\left(E_{y}[\Psi]\right)>V_{y}[\Psi]
$$

It is important to note that in this model the concept of risk aversion is not independent of the distribution itself - this is not the case in EUT where the degree of risk aversion can be determined solely from the utility functions of different individuals.

All of these cases will also hold for the subjective risk premium if the objective expected value $E_{y}[\Psi]$ is replaced everywhere by the subjective counterpart $E_{y}^{s}[\Psi]$.

Using the distinction between the objective risk premium $r^{\Psi}$ and the subjective (Pratt-Arrow) risk premium $r_{S}^{\Psi}$ we can calculate the degree to which the decision maker's beliefs about their own risk aversion differ from their actual risk-aversion when compared to the true distribution. We term this the Subjectivity Premium $r_{\pi}^{\Psi}$

$$
r_{\pi}^{\Psi}=r^{\Psi}-r_{S}^{\Psi}=E_{y}[\Psi]-E_{y}^{s}[\Psi]=\int x f(x) d x-\int x m(x) f(x) d x
$$

It is important to note that $r_{\pi}^{\Psi}$ does not answer the question of how introducing decision weights to the model affects the risk premium. This needs to be done by comparing either $r^{\Psi}$ or $r_{S}^{\Psi}$, when calculated using probability transformations, to the situation where there are no 
probability transformations at all (i.e., when evaluating the prospect using $V_{y}[\Psi]$ with $m_{-}$and $m_{+}$equal to 1 for all $x$ ). In deriving any of the three premia given above, the value of the prospect $V_{y}[\Psi]$ always incorporates decision weights rather than objective probabilities. The distinction is rather whether the risk premium related to this value is relative to the actual expected value of the prospect, or to the decision maker's subjective assessment of this expected value. That is, not whether the risk premium is affected by probability distortions, but whether the risk attitude is that which the subject perceives herself to hold, or that which would be observed by an external observer.

\section{Approximate Solutions}

Whilst (5) is the exact equation for the risk premium for a particular prospect, solutions can only be obtained for certain specifications of the utility function and probability distribution. Following Pratt (1964) we can make more general statements about the shape of these functions if we restrict our analysis to "small prospects", that is, those with extremely small variance. This enables us to examine local risk attitude at a specific point. Initially, we restrict our analysis to local risk attitude at the reference point itself. In other words we are interested in the risk attitude to a small prospect with an expected value of zero relative to the reference point.

In what follows we first explore these issues in the simpler world where probability is always treated objectively $\left(m_{-}=m_{+}=1\right)$. Let $\Psi_{\varepsilon}$ denote the prospect formed by a random variable of outcomes with $E_{y}\left[\Psi_{\varepsilon}\right]=E[\varepsilon]=0$ and infinitesimally small variance such that $E\left[\varepsilon^{2}\right]$ is negligible. This can be extended to the more general cases, and we examine the case where the small prospect is not centred on the reference point (i.e., where $E[\varepsilon] \neq 0$ ) in section 3.1.2 below.

Thereafter we reintroduce subjective probability weighting into the analysis. We first discuss the results of Levy and Levy in relationship to the subjective (Pratt-Arrow) risk premium and decision weights in section 3.2.1, we then extend their analysis to include the distinction between different utility curvatures for gains and losses, and the concept of loss aversion in the same way as we had previously analysed in the objective cases in 3.2.2. This analysis is completed for both the objective and subjective risk premia. 


\subsection{Risk Aversion with No Probability Distortion}

\subsubsection{At Reference Point}

Case 1 From the above definition of the risk premium, if $E[\varepsilon]-r^{\Psi}>0$ then the individual is risk seeking for this gamble. Thus, denoting the probability of a gain by $p$, the risk premium is defined by:

$$
\begin{aligned}
v_{+}\left(E[\varepsilon]-r^{\Psi}\right) & =(1-p) \lambda E_{y}\left[v_{-}(\varepsilon) \mid \varepsilon<0\right]+p E_{y}\left[v_{+}(\varepsilon) \mid \varepsilon>0\right] \\
v_{+}\left(-r^{\Psi}\right) & =(1-p) \lambda E_{y}\left[v_{-}(\varepsilon) \mid \varepsilon<0\right]+p E_{y}\left[v_{+}(\varepsilon) \mid \varepsilon>0\right]
\end{aligned}
$$

Taking a first order Taylor approximation around the reference point (i.e., around 0) on the left hand side of the equation yields

$$
L H S \approx v_{+}(0)-v_{+}^{\prime}(0) r^{\Psi}
$$

And a second order approximation around the reference point on the right hand side gives

$$
\begin{aligned}
R H S \approx & E_{y}\left[v_{-}(0)+v_{-}^{\prime}(0)(\varepsilon \mid \varepsilon<0)+\frac{1}{2} v_{-}^{\prime \prime}(0)\left(\varepsilon^{2} \mid \varepsilon<0\right)\right] \lambda(1-p) \\
& +E_{y}\left[v_{+}(0)+v_{+}^{\prime}(0)(\varepsilon \mid \varepsilon>0)+\frac{1}{2} v_{+}^{\prime \prime}(0)\left(\varepsilon^{2} \mid \varepsilon>0\right)\right] p
\end{aligned}
$$

Because $v(0)=0(? ?), L H S \approx R H S$ implies

$$
\begin{aligned}
-v_{+}^{\prime}(0) r^{\Psi} \approx & \left(v_{-}^{\prime}(0) E_{y}[\varepsilon \mid \varepsilon<0]+\frac{1}{2} v_{-}^{\prime \prime}(0) E_{y}\left[\varepsilon^{2} \mid \varepsilon<0\right]\right) \lambda(1-p) \\
& +\left(v_{+}^{\prime}(0) E_{y}[\varepsilon \mid \varepsilon>0]+\frac{1}{2} v_{+}^{\prime \prime}(0) E_{y}\left[\varepsilon^{2} \mid \varepsilon>0\right]\right) p \\
r^{\Psi} \approx & -\left(\begin{array}{c}
\left(\frac{v_{-}^{\prime}(0)}{v_{+}^{\prime}(0)} E_{y}[\varepsilon \mid \varepsilon<0]+\frac{v_{-}^{\prime \prime}(0)}{2 v_{+}^{\prime}(0)} E_{y}\left[\varepsilon^{2} \mid \varepsilon<0\right]\right) \lambda(1-p) \\
+\left(E_{y}[\varepsilon \mid \varepsilon>0]+\frac{v_{+}^{\prime \prime}(0)}{2 v_{+}^{\prime}(0)} E_{y}\left[\varepsilon^{2} \mid \varepsilon>0\right]\right) p
\end{array}\right)
\end{aligned}
$$


Since $\varepsilon$ is a zero-mean random variable, $E_{y}[\varepsilon \mid \varepsilon<0](1-p)=-E_{y}[\varepsilon \mid \varepsilon>0] p$, which allows this to be further simplified to

$$
\begin{aligned}
r^{\Psi} \approx & E_{y}[\varepsilon \mid \varepsilon>0] p\left(\frac{v_{-}^{\prime}(0)}{v_{+}^{\prime}(0)} \lambda-1\right) \\
& -\frac{1}{2}\left(\frac{v_{-}^{\prime \prime}(0)}{v_{+}^{\prime}(0)} \lambda E_{y}\left[\varepsilon^{2} \mid \varepsilon<0\right](1-p)+\frac{v_{+}^{\prime \prime}(0)}{v_{+}^{\prime}(0)} E_{y}\left[\varepsilon^{2} \mid \varepsilon>0\right] p\right)
\end{aligned}
$$

Furthermore, if we follow the suggestion of Köbberling and Wakker (2003) that we adopt a scaling convention for loss aversion such that the index of loss aversion $\lambda$ is captured by the ratio of the slope of the utility function from below at $0\left(v_{\uparrow}^{\prime}(0)\right)$ to the slope of the utility function from above at $0\left(v_{\downarrow}^{\prime}(0)\right)$, then we must have,

$$
\begin{aligned}
\lambda & =\frac{v_{\uparrow}^{\prime}(0)}{v_{\downarrow}^{\prime}(0)} \\
\lambda & =\frac{\lambda v_{-}^{\prime}(0)}{v_{+}^{\prime}(0)}, \text { and so, } \\
v_{-}^{\prime}(0) & =v_{+}^{\prime}(0)=v^{\prime}(0)
\end{aligned}
$$

This places restrictions on the permissible forms of $v(x)$, but has a number of advantages in that the risk attitude due to loss aversion in mixed prospects can be precisely separated from that due to utility curvature in gains-only or loss-only prospects in a way that is independent of the choice of unit. Using this convention, (17) becomes

$$
\begin{aligned}
r^{\Psi} \approx & (\lambda-1) p E_{y}[\varepsilon \mid \varepsilon>0] \\
& -\frac{1}{2}\left(\frac{v_{-}^{\prime \prime}(0)}{v^{\prime}(0)} \lambda(1-p) E_{y}\left[\varepsilon^{2} \mid \varepsilon<0\right]+\frac{v_{+}^{\prime \prime}(0)}{v^{\prime}(0)} p E_{y}\left[\varepsilon^{2} \mid \varepsilon>0\right]\right)
\end{aligned}
$$

Case 2 Similarly, if $E_{y}[\Psi]-r^{\Psi}<0$, then $r^{\Psi}>0, L H S \approx-\lambda v_{-}^{\prime}(0) r^{\Psi}$ and the second order approximation for RHS remains the same (15). Thus 


$$
\begin{aligned}
r^{\Psi} \approx & -\left(\begin{array}{c}
\left(E_{y}[\varepsilon \mid \varepsilon<0]+\frac{v_{-}^{\prime \prime}(0)}{2 v_{-}^{\prime}(0)} E_{y}\left[\varepsilon^{2} \mid \varepsilon<0\right]\right)(1-p) \\
+\frac{1}{\lambda}\left(\frac{v_{+}^{\prime}(0)}{v_{-}^{\prime}(0)} E_{y}[\varepsilon \mid \varepsilon>0]+\frac{v_{+}^{\prime \prime}(0)}{2 v_{-}^{\prime}(0)} E_{y}\left[\varepsilon^{2} \mid \varepsilon>0\right]\right) p
\end{array}\right) \\
r^{\Psi} \approx & E_{y}[\varepsilon \mid \varepsilon>0] p\left(1-\frac{v_{+}^{\prime}(0)}{\lambda v_{-}^{\prime}(0)}\right) \\
& -\frac{1}{2}\left(\frac{v_{-}^{\prime \prime}(0)}{v_{-}^{\prime}(0)} E_{y}\left[\varepsilon^{2} \mid \varepsilon<0\right](1-p)+\frac{v_{+}^{\prime \prime}(0)}{\lambda v_{-}^{\prime}(0)} E_{y}\left[\varepsilon^{2} \mid \varepsilon>0\right] p\right)
\end{aligned}
$$

And with Köbberling and Wakker's index of loss aversion:

$$
\begin{aligned}
r^{\Psi} \approx & \left(1-\frac{1}{\lambda}\right) p E_{y}[\varepsilon \mid \varepsilon>0] \\
& -\frac{1}{2}\left(\frac{v_{-}^{\prime \prime}(0)}{v^{\prime}(0)}(1-p) E_{y}\left[\varepsilon^{2} \mid \varepsilon<0\right]+\frac{v_{+}^{\prime \prime}(0)}{\lambda v^{\prime}(0)} p E_{y}\left[\varepsilon^{2} \mid \varepsilon>0\right]\right)
\end{aligned}
$$

Implications Examining these results enables us to give the sufficient conditions on the shape of the utility functions and on the loss aversion parameter to guarantee risk aversion for small prospects. These results are analogous to the condition of concavity of the utility function given by Pratt and Arrow for the more simple case where utility is attributed to total wealth rather than changes in wealth. These conditions are summarised in the propositions below.

Proposition 1 If we have a monotonically increasing utility function

$$
\begin{aligned}
& v(\varepsilon)=\left\{\begin{array}{c}
v_{+}(\varepsilon), \text { if } \varepsilon>0 \\
\lambda v_{-}(\varepsilon), \text { if } \varepsilon \leq 0
\end{array}\right. \\
& v(0)=0
\end{aligned}
$$

and face a gamble with outcomes distributed according a random variable $\varepsilon$ representing deviations from the reference point $y$. The gamble has zero mean, $E_{y}\left[\Psi_{\varepsilon}\right]=E(\varepsilon)=0$ and infinitessimal variance, and

a)

$$
\lambda>1
$$


b)

$$
v_{+}^{\prime \prime}(0)<0 \text { and } v_{-}^{\prime \prime}(0)<0
$$

then the risk premium is positive, $r^{\Psi}>0$ (i.e., the individual is locally risk-averse for small changes in wealth)

Proof. Insert (23) and (24) into (21). $p$, and $E_{y}[\varepsilon \mid \varepsilon>0]$, are greater than zero, and $v_{+}^{\prime}(0)$, and $v_{-}^{\prime}(0)$ are also positive since $v(\varepsilon)$ is monotonically increasing. Thus (23) is sufficient to ensure that the first term is positive. In addition, since $(1-p), E_{y}\left[\varepsilon^{2} \mid \varepsilon<0\right]$, and $E_{y}\left[\varepsilon^{2} \mid \varepsilon>0\right]$ are positive, (24) is sufficient to ensure that the second term in brackets is negative, guaranteeing that the expressions as a whole will be positive.

This violates the premise of Case 1 that $r^{\Psi}<0$, but allows that of Case 2 that $r^{\Psi}>0$. Thus, these conditions are sufficient to ensure that the individual will pay to avoid a fair gamble (with respect to the reference point) for small changes to expected wealth from the reference point.

The terms on the right hand sides of (19) and (21) are analogous to the Pratt-Arrow risk premium. In the standard Pratt-Arrow framework, concavity of the utility function is sufficient to ensure risk aversion and thereby a positive risk premium. With the addition of a reference point to divide outcomes into gains or losses, however, the first terms in these equations also need to be positive and this condition is neither necessary nor sufficient. To ensure local risk aversion for a small prospect it must also be the case that loss aversion exists (making the first term positive).

Loss aversion is central to Kahneman and Tversky's Prospect Theory and has a great deal of empirical support. Köbberling and Wakker motivate their proposed index of loss aversion, $\lambda=\frac{v_{\uparrow}^{\prime}(0)}{v_{\downarrow}^{\prime}(0)}$, by pointing out that this measure allows us to define loss aversion independently of the unit of payment. This is not true of the most common version of CPT due to Tversky and Kahneman (1992) where they assume that $v_{+}(x)=x^{\alpha}$ for gains and $v_{-}(x)=-|-x|^{\beta}$ for losses. With this formulation $v(1)=-v(-1)=1$, which implies a scaling convention such that $\lambda=\frac{-v(-1)}{v(1)}$. This convention is not independent of the unit of payment. Both measures allow loss aversion to be neatly separated from the attribution of basic utility to gains and 
losses independently. In particular Köbberling and Wakker show that these measures allow us to characterise individuals with identical preferences for all gains-only and loss-only prospects whilst differing in their preferences for mixed prospects. However, in the class of measures that have this property, only the ratio of slopes at the origin is independent of the unit chosen for payment. Our analysis provides another reason for this measure: the ratio arises naturally in the approximate solutions for the risk premium (Equations (21) and (19)), and choosing this as our definition of loss aversion enables us to simply and elegantly express the effect of loss aversion on the risk premium. That is, in proposition 1 , our scaling convention for loss aversion enables condition a) to be $\lambda>1$, rather than $\lambda>\frac{v_{+}^{\prime}(0)}{v_{-}^{\prime}(0)}$.

This definition of loss aversion, whilst possessing a number of desirable properties, does imply some restrictions on the parametric forms that can be taken by the functions assigning basic utility, $v_{+}(x)$ and $v_{-}(x)$. In particular, as discussed by Köbberling and Wakker, this form of the loss aversion index implies considerable difficulties for the commonly utilised constant relative risk aversion (CRRA) power utility functions. These forms have zero or infinite derivatives at zero if the power is not one. This means that unless the curvature parameter for gains is equal to that for losses, then the loss aversion index is either zero or infinite and it is impossible to define a basic utility function independently of loss aversion. They demonstrate, however, that constant absolute risk aversion (CARA) utility functions (i.e., exponential) do not face these problems at the reference point.

The effect of loss aversion on the risk premium is clear, it increases the risk premium. However, another central intuition behind Prospect Theory, namely diminishing sensitivity to outcomes from the reference point, has an indeterminate effect on the risk premium. This intuition requires that the utility function is concave above the reference point, but convex below. Thus decision makers are risk averse to gambles with only gains outcomes, and risk-seeking for lossonly gambles. This makes the sign of the Pratt-Arrow part of the risk premium indeterminate when combined with the notion of diminishing sensitivity.

Notice, too, that this proposition may be derived using either Case 1 or Case 2. However, if conditions a) and b) hold and the decision maker is thus locally risk averse, then the actual value of the risk premium is determined from (21) in Case 2 where $r^{\Psi}>0$. Since these conditions are sufficient, but not necessary there may be instances where they do not hold but where the risk 
premium is positive.

The sufficiency conditions for risk-seeking are derived similarly,

Proposition 2 If we have a monotonically increasing utility function

$$
\begin{aligned}
& v(\varepsilon)=\left\{\begin{array}{c}
v_{+}(\varepsilon), \text { if } \varepsilon>0 \\
\lambda v_{-}(\varepsilon), \text { if } \varepsilon \leq 0
\end{array}\right. \\
& v(0)=0
\end{aligned}
$$

and face a gamble with outcomes distributed according a random variable $\varepsilon$ representing deviations from the reference point $y$. The gamble has zero mean, $E_{y}\left[\Psi_{\varepsilon}\right]=E(\varepsilon)=0$ and infinitessimal variance, and

c)

$$
\lambda<1
$$

d)

$$
v_{+}^{\prime \prime}(0)>0 \text { and } v_{-}^{\prime \prime}(0)>0
$$

then $r^{\Psi}<0$ and the risk premium is negative (i.e., the individual is locally risk-loving for small changes in wealth)

Proof. As for Proposition 1

We can further strengthen condition b) if in addition to being an actuarially fair gamble, $E[\varepsilon]=0$, we also assume that the following symmetry holds over the distribution: $p E\left[\varepsilon^{2} \mid \varepsilon>0\right]=$ $(1-p) E\left[\varepsilon^{2} \mid \varepsilon<0\right]^{1}$ In this case 19 simplifies to:

$$
\begin{aligned}
r^{\Psi} \approx & \left(\lambda^{2}-1\right) p E_{y}[\varepsilon \mid \varepsilon>0] \\
& -\frac{1}{2}\left(\frac{v_{-}^{\prime \prime}(0)}{v_{+}^{\prime}(0)} \lambda(1-p) E_{y}\left[\varepsilon^{2} \mid \varepsilon<0\right]+\frac{v_{+}^{\prime \prime}(0)}{v_{+}^{\prime}(0)} p E_{y}\left[\varepsilon^{2} \mid \varepsilon>0\right]\right) \\
r^{\Psi} \approx & \left(\lambda^{2}-1\right) p E_{y}[\varepsilon \mid \varepsilon>0]-\frac{\lambda v_{-}^{\prime \prime}(0)+v_{+}^{\prime \prime}(0)}{2 v_{+}^{\prime}(0)} p E_{y}\left[\varepsilon^{2} \mid \varepsilon>0\right]
\end{aligned}
$$


and condition b) can be strengthened to

$$
\lambda v_{-}^{\prime \prime}(0)+v_{+}^{\prime \prime}(0)<0
$$

Proposition 3 If we have a monotonically increasing utility function

$$
\begin{aligned}
& v(\varepsilon)=\left\{\begin{array}{c}
v_{+}(\varepsilon), \text { if } \varepsilon>0 \\
\lambda v_{-}(\varepsilon), \text { if } \varepsilon \leq 0
\end{array}\right. \\
& v(0)=0
\end{aligned}
$$

and face a gamble with outcomes distributed according a random variable $\varepsilon$ representing deviations from the reference point $y$. The gamble has zero mean, $E_{y}\left[\Psi_{\varepsilon}\right]=E(\varepsilon)=0$, infinitessimal variance, and symmetry around 0 in the sense that $p E\left[\varepsilon^{2} \mid \varepsilon>0\right]=(1-p) E\left[\varepsilon^{2} \mid \varepsilon<0\right]$, and e)

$$
\lambda>1
$$

f)

$$
\lambda v_{-}^{\prime \prime}(0)+v_{+}^{\prime \prime}(0)<0
$$

then the risk premium is positive, $r^{\Psi}>0$ (i.e., the individual is locally risk-averse for small changes in wealth)

Condition $\mathrm{f}$ ) is a less onerous condition than b) as it does not require that both $v_{-}^{\prime \prime}(0)$ and $v_{+}^{\prime \prime}(0)$ are locally concave, but rather that any local convexity must be outweighed by local concavity after taking loss aversion into account.

Similarly, Proposition 2 becomes 
Proposition 4 If we have a monotonically increasing utility function

$$
\begin{aligned}
& v(\varepsilon)=\left\{\begin{array}{c}
v_{+}(\varepsilon), \text { if } \varepsilon>0 \\
\lambda v_{-}(\varepsilon), \text { if } \varepsilon \leq 0
\end{array}\right. \\
& v(0)=0
\end{aligned}
$$

and face a gamble with outcomes distributed according a random variable $\varepsilon$ representing deviations from the reference point $y$. The gamble has zero mean, $E_{y}\left[\Psi_{\varepsilon}\right]=E(\varepsilon)=0$, infinitessimal variance, and symmetry around 0 in the sense that $p E\left[\varepsilon^{2} \mid \varepsilon>0\right]=(1-p) E\left[\varepsilon^{2} \mid \varepsilon<0\right]$, and c)

$$
\lambda<1
$$

d)

$$
\lambda v_{-}^{\prime \prime}(0)+v_{+}^{\prime \prime}(0)>0
$$

then $r^{\Psi}<0$ and the risk premium is negative (i.e., the individual is locally risk-loving for small changes in wealth)

Standard Pratt-Arrow Risk Premium as a Special Case In standard Expected Utility Theory, absolute risk aversion is measured by the Pratt-Arrow coefficient of absolute risk aversion, which at $x$ is defined as $r_{A}(x)=-v^{\prime \prime}(x) / v^{\prime}(x)$. When measured at $x=0$ the Pratt-Arrow measure is related to a special case of the risk premium as described above. Specifically, if the following three conditions hold, the risk premium has a one-to-one relation to the coefficient of risk aversion measured at the reference point:

Condition $5 v_{\downarrow}^{\prime}(0)=v_{\uparrow}^{\prime}(0)=v^{\prime}(0)$, that $i s, \lambda=1$ (The slope of the utility function at the reference point is identical for small gains and losses)

Condition $6 v_{+}^{\prime \prime}(0)=\lambda v_{-}^{\prime \prime}(0)=v^{\prime \prime}(0)$ (The curvature of the utility function at the reference point is identical for gains and losses) 
Condition $7 E\left[\varepsilon^{2} \mid \varepsilon>0\right] p=E\left[\varepsilon^{2} \mid \varepsilon<0\right](1-p)=\frac{\sigma^{2}}{2}$ (The outcomes are symmetrical about the reference point - thus $p=\frac{1}{2}$ )

With these conditions we can specialise (19) and (21) to obtain,

$$
\begin{aligned}
r^{\Psi} & =-\frac{v^{\prime \prime}(0)}{2 v^{\prime}(0)} \sigma^{2} \\
& =r_{A}(0) \frac{\sigma^{2}}{2}
\end{aligned}
$$

Thus, for any given distribution the higher the aversion to risk, the greater the risk premium required for the individual to take the gamble. This is the classical result of Pratt and Arrow.

\subsubsection{Local Risk Aversion Away from the Reference Point}

The discussion above centres on local risk aversion for small prospects at the reference point (i.e., with $E[\varepsilon]=0$ ). However, this is a special case and we need to be able to examine local risk premia for points that are distinct from the reference point. For example, what conditions on the utility function are sufficient for risk aversion when evaluating a gamble with a positive expected return relative to the reference point?

$$
v\left(E[\varepsilon]-r^{\Psi}\right)=E_{y}[v(\varepsilon)]
$$

Unlike the situation where the expected value of the random variable is equal to the reference point, the RHS of this expression uses only $v_{+}(\varepsilon)$ or $v_{-}(\varepsilon)$ to evaluate the random variable. This is because, with infinitessimal variance a positive mean implies that all outcomes will be positive, whereas with a negative mean all outcomes are negative.

Taking a first order Taylor approximation around $E[\varepsilon]$ on the left hand side yields

$$
\begin{aligned}
L H S & \approx v(E[\varepsilon])+\left(\left(E[\varepsilon]-r^{\Psi}\right)-E[\varepsilon]\right) v^{\prime}(E[\varepsilon]) \\
& \approx v(E[\varepsilon])-r^{\Psi} v^{\prime}(E[\varepsilon])
\end{aligned}
$$


And a second order approximation around $E[\varepsilon]$ on the right hand side gives

$$
R H S \approx E_{y}\left[v(E[\varepsilon])+v^{\prime}(E[\varepsilon])(\varepsilon-E[\varepsilon])+\frac{1}{2} v^{\prime \prime}(E[\varepsilon])(\varepsilon-E[\varepsilon])^{2}\right]
$$

$L H S \approx R H S$ implies

$$
\begin{aligned}
v(E[\varepsilon])-r^{\Psi} v^{\prime}(E[\varepsilon]) \approx & v(E[\varepsilon])+v^{\prime}(E[\varepsilon]) E_{y}[(\varepsilon-E[\varepsilon])] \\
& +\frac{1}{2} v^{\prime \prime}(E[\varepsilon]) E_{y}\left[(\varepsilon-E[\varepsilon])^{2}\right] \\
r^{\Psi} \approx & -E_{y}[(\varepsilon-E[\varepsilon])]-\frac{1}{2} \frac{v^{\prime \prime}(E[\varepsilon])}{v^{\prime}(E[\varepsilon])} E_{y}\left[(\varepsilon-E[\varepsilon])^{2}\right] \\
r^{\Psi} \approx & -\frac{1}{2} \frac{v^{\prime \prime}(E[\varepsilon])}{v^{\prime}(E[\varepsilon])} \sigma^{2}
\end{aligned}
$$

Again, there are two important cases here. If $E[\varepsilon]<0$, then this is evaluated as

$$
r^{\Psi} \approx-\frac{1}{2} \frac{v_{-}^{\prime \prime}(E[\varepsilon])}{v_{-}^{\prime}(E[\varepsilon])} \sigma^{2}
$$

whereas if the expected value is positive, then the gains functions are used:

$$
r^{\Psi} \approx-\frac{1}{2} \frac{v_{+}^{\prime \prime}(E[\varepsilon])}{v_{+}^{\prime}(E[\varepsilon])} \sigma^{2}
$$

Note that in the first of these equations the loss aversion parameters on the top and bottom cancel out. This reflects the fact that the evaluation is entirely in losses, and therefore loss aversion has no effect (it only affects decision making for mixed prospects).

This result is exactly analogous to the Pratt-Arrow risk premium, except for the specification that the value function is defined for gains or losses as appropriate. Thus the individual is locally risk averse if and only if the utility function for gains is concave at that point. Note that the prospect is being evaluated from the reference point which is distinct from the expected outcome.

So, for small prospects it is only at the reference point that the sign of the local risk premium is dependent on both the gains and loss utility functions. It is also important that, at this point it is not only the curvature of these functions that matters, but also the ratio of the slopes. Lastly, unlike the Pratt-Arrow risk premium, in reference dependent utility, constraints on the value 
function are no longer both necessary and sufficient to determine the sign of the risk premium the constraints given are sufficient, but not necessary.

\subsection{Non-Linear Probability Weighting}

\subsubsection{Previous Analysis - Levy and Levy}

Arrow's Approach Levy and Levy (2002) investigate a restricted version of this problem using the two differing approaches of Arrow (1965) and Pratt (1964). Arrow's approach considers a bet with only two outcomes of $+h$ and $-h$, where $h$ is vanishingly small. In our analysis we have used Pratt's more general approach where a distribution with infinitessimal variance is considered and, with the exception of a brief discussion here of Levy and Levy's results using the Arrow approach, will continue to employ Pratt's methodology in the remainder of the paper.

Arrow's original risk premium refers to the increase in probability above $50 \%$ for the positive value of the bet, $h$, that is required to induce the decision maker to accept the bet. Thus, it is the value of $p-\frac{1}{2}$ where $p=\frac{1}{2}$ is risk neutrality and $p>(<) \frac{1}{2}$ represents risk aversion (risk seeking). Arrow showed that $p-\frac{1}{2} \approx-\frac{v^{\prime \prime}(0)}{v^{\prime}(0)} \frac{h}{4}$. The risk premium in this context relates to the amount of increase in the expected value of the bet represented by the prospect $(-h, 1-p ; h, p)$ over that of the fair bet $(-h, 50 \% ; h, 50 \%)$. Thus, the higher the value of $p$, required to induce the decision maker to take the bet, the greater the expected utility the decision maker will forego to avoid the gamble.

Levy and Levy investigate only the role of decision weighting on the risk premium, and do not distinguish between potential changes in the curvature of the utility function above and below the reference point, nor do they incorporate loss aversion into their analysis. The primary conclusion of employing Arrow's approach is that the probability distortion induced by CPT "systematically increases Arrow's risk premium", which is defined as $p_{0}-\frac{1}{2}$, where $p_{0}$ is the solution to

$$
w^{*+}\left(p_{0}\right)-w^{*-}\left(1-p_{0}\right) \approx-\frac{v^{\prime \prime}(W)}{v^{\prime}(W)} \frac{h}{2}
$$

and $W$ is current wealth ${ }^{2}$. That is, they show that $p_{0}>p$.

Some aspects of their analysis require further comment. Firstly, if current wealth is taken to 
be the reference point, (39) becomes

$$
w^{*+}\left(p_{0}\right)-w^{*-}\left(1-p_{0}\right) \approx-\frac{v^{\prime \prime}(0)}{v^{\prime}(0)} \frac{h}{2}
$$

This requires either that the utility function is continuous and differentiable at 0 , or that further analysis is made to define whether the derivatives of the utility function are taken from the left or the right of the reference point. Levy and Levy do not incorporate reference dependent utility functions (though they do make use of a distinction between gains and losses), and thus implicitly make the first assumption.

Secondly their solution for the Arrow risk premium requires that the decision weights are normalised so that they add up to unity. This is only required if $W$ is taken to be at the reference point. If this is not the case the small prospect will consist entirely of gains or entirely of losses, and the decision weights will always sum to one, so we infer that they intend the analysis to apply at a reference point. For this simple binary prospect the normalisation requires that the adjusted weights used in (39) are derived from the unadjusted CPT weights, $w^{+}(p)$ and $w^{-}(1-p)$, by $w^{*+}\left(p_{0}\right)=w^{+}(p) /\left(w^{+}(p)+w^{-}(1-p)\right)$ and $w^{*-}(p)=w^{-}(p) /\left(w^{+}(p)+w^{-}(1-p)\right)$. As a rationale for this move they argue in a note that Tversky and Kahneman (1992) propose this normalisation in order to overcome technical drawbacks of the original Prospect Theory (Kahneman and Tversky 1979). A reading of the original text reveals that Tversky and Kahneman do indeed allow that this normalisation can be effective in overcoming problems of the original Prospect Theory, but argue that this can be done better through using the alternative rank-dependent functional in Cumulative Prospect Theory. CPT replaces the need to normalise the probabilities to unity, and such a normalisation is not supported within CPT. I quote the relevant passage in full:

These problems [in 1979 Prospect Theory] can be handled by assuming that transparently dominated prospects are eliminated in the editing phase, and by normalizing the weights so they add to unity. Alternatively, both problems can be solved by the rank-dependent or cumulative functional.

On the very next page they state that "For mixed prospects, however, the sum can be either 
smaller or greater than 1, because the decision weights for gains and for losses are defined by separate capacities."

The "normalisation" employed by Levy and Levy cannot be justified within the framework of CPT, but requires additional justification, which has not been provided by Tversky and Kahneman. In fact, because adjusting the decision weights in this way changes the CPT valuations given to mixed prospects (though not to gains-only or loss-only prospects for which the decision weights will always sum to unity) it may also change the preference ranking between prospects and thus is not a neutral "normalisation" at all.

To demonstrate that this may occur, consider the choice between the prospect

$$
\mathbf{f}=(-100,25 \% ; 100 ; 75 \%)
$$

and the prospect

$$
\mathbf{g}=(-50,95 \% ; 550,5 \%)
$$

These choice of values have been chosen to provide a simple example of where the normalisation employed by Levy and Levy might lead to a switch in the predicted preference ordering in CPT even for binary prospects. For simplicity I assume a linear utility function, so the utility of the gains and losses in the two prospects may be utilised directly. This is without loss of generality as one could always determine the monetary outcomes required to produce the appropriate utility figures by taking the inverse of any (reference dependent and loss averse) utility function. I use the weighting functions used by Levy and Levy (that is, those suggested by Tversky and Kahneman 1992):

$$
\begin{aligned}
& w^{-}(p)=\frac{p^{\delta}}{\left(p^{\delta}+(1-p)^{\delta}\right)^{\frac{1}{\delta}}} \\
& w^{+}(p)=\frac{p^{\gamma}}{\left(p^{\gamma}+(1-p)^{\gamma}\right)^{\frac{1}{\gamma}}}
\end{aligned}
$$

where $p$ is a cumulative probability for losses, and a decumulative probability for gains, and $\delta=0.69, \gamma=0.61$. 
With these assumptions $f$ is evaluated as:

$$
\begin{aligned}
V(\mathbf{f}) & =-100\left(-w^{-}(0)\right)+100\left(-w^{+}(0)\right) \\
& =-100 w^{-}(25 \%)+100 w^{+}(75 \%)
\end{aligned}
$$

Similarly $g$ is:

$$
V(\mathbf{g})=-50 w^{-}(95 \%)+550 w^{+}(5 \%)
$$

The unadjusted decision weights are $w^{-}(25 \%)=0.294, w^{-}(95 \%)=0.850, w^{+}(75 \%)=$ $0.568, w^{+}(5 \%)=0.132$, and the "normalised" weights are: $w^{*-}(25 \%)=0.341, w^{*-}(95 \%)=$ $0.866, w^{*+}(75 \%)=0.659, w^{*+}(5 \%)=0.134$. Note that the transformation has a comeasurately larger effect on the probability weight used in $\mathbf{f}$, than in $\mathbf{g}$.

The resulting CPT evaluations of the two prospects before normalisation are $V(\mathbf{f})=27.475$, and $V(\mathbf{g})=29.900$. The decision maker would thus choose $\mathbf{g}$. However, using the adjusted decision weights we obtain $V^{*}(\mathbf{f})=31.881$, and $V^{*}(\mathbf{g})=30.463$ : in this case the decision maker would choose prospect $\mathbf{f}$. This demonstrates that the adjustment used by Levy and Levy is not neutral with regard to preference ordering, and is thus neither a normalisation, nor justified.

In and endnote to their paper they provide the equivalent result to (39) where the decision weight adjustment is not employed:

$$
\begin{aligned}
w^{+}\left(p_{0}\right)-w^{-}\left(1-p_{0}\right) \approx & -\frac{v^{\prime \prime}(0)}{v^{\prime}(0)} \frac{h}{2}\left[w^{+}\left(p_{0}\right)+w^{-}\left(1-p_{0}\right)\right] \\
& -\frac{v(0)}{v^{\prime}(0)} \frac{1}{h}\left[w^{+}\left(p_{0}\right)+w^{-}\left(1-p_{0}\right)-1\right] \\
w^{+}\left(p_{0}\right)-w^{-}\left(1-p_{0}\right) \approx & -\frac{v^{\prime \prime}(0)}{v^{\prime}(0)} \frac{h}{2}\left[w^{+}\left(p_{0}\right)+w^{-}\left(1-p_{0}\right)\right] \\
\frac{w^{+}\left(p_{0}\right)-w^{-}\left(1-p_{0}\right)}{w^{+}\left(p_{0}\right)+w^{-}\left(1-p_{0}\right)} \approx & -\frac{v^{\prime \prime}(0)}{v^{\prime}(0)} \frac{h}{2}
\end{aligned}
$$

The second line follows from the fact that in $\operatorname{CPT} v(0)=0$ (a valid normalisation within the theory due to the non-uniqueness of the utility function to an affine transformation). Away from the reference point the small prospect used by Arrow lies entirely in gains or in losses and the decision weights thus sum to unity resulting in Levy and Levy's original formula, (39). 
Although the changes required by the non-validity of the decision weight adjustments require an adjustment to (40), the result they report remains unchanged. That is, for the domain of risk aversion (where $p>\frac{1}{2}$ ) the CPT probability distortion systematically increases Arrow's risk premium. However, even in their original analysis this was not true where Arrow's risk premium was negative - here the probability distortion could either increase or decrease the risk premium. In addition, their result is very dependent on the precise values used for $\delta$ and $\gamma$.

Both these points can be illustrated using the graphical analysis of Levy and Levy. Recall that the Arrow risk premium is defined as $p-\frac{1}{2} \approx-\frac{v^{\prime \prime}(0)}{v^{\prime}(0)} \frac{h}{4}$, or equivalently $2 p-1 \approx-\frac{v^{\prime \prime}(0)}{v^{\prime}(0)} \frac{h}{2}$. The value of $p$ for which this is approximately true may be compared directly to the value of $p_{0}$ which solves (41). If $p_{0}$ is required to be greater than $p$ to give a particular value of $-\frac{v^{\prime \prime}(0)}{v^{\prime}(0)} \frac{h}{2}$, then the CPT distortion increases Arrow's risk premium. That is, the decision maker is more risk averse, or less risk seeking as appropriate. Figure 1 graphs $2 p-1$ and $\frac{w^{+}\left(p_{0}\right)-w^{-}\left(1-p_{0}\right)}{w^{+}\left(p_{0}\right)+w^{-}\left(1-p_{0}\right)}$ for all values of $p$ and $p_{0}$.

\section{Insert Figure 1 here}

As can be seen from the figure, $p_{0}>p$ for all cases where Arrow's risk premium is positive. This is the conclusion of Levy and Levy. For decision makers close to risk neutrality (near $\left.-\frac{v^{\prime \prime}(0)}{v^{\prime}(0)} \frac{h}{2}=0\right)$ there is an area where $p<\frac{1}{2}$, but $p_{0}>\frac{1}{2}$. Here probability distortion causes a mild risk seeker to be become mildly risk averse. However, where decision makers are sufficiently risk seeking, CPT decision weights actually decrease the risk premium and make the decision maker more strongly risk seeking.

In Figure 1 we have employed the same values of $\delta=0.69$, and $\gamma=0.61$ as used by Levy and Levy. However, Figure 2 shows that their conclusion is very sensitive to these values. Reversing the the values for gains and losses (to $\delta=0.61$, and $\gamma=0.69$ ) means that their conclusion no longer holds and, for low levels of Arrow risk aversion, CPT decision weights actually decrease the risk premium. In fact, Levy and Levy's conclusion holds if and only if $\delta \geq \gamma$. The values they utilised were the median estimates on empirical data by Tversky and Kahneman. Subsequent investigation on the decision weighting function has shown that, whilst this values do fit median data relatively well, there is a very broad range of individual variation and that $\delta \geq \gamma$ certainly cannot be taken for granted. (Abdellaoui 2000, Gonzalez and Wu 1999, Wu and Gonzalez 1996, 1999). 


\section{Insert Figure 2}

Pratt's Approach Levy and Levy also analyse Pratt's approach to the risk premium, using small distributions of outcomes as we have done in Section 3 above. Again abstracting from issues of reference dependence and loss aversion, they seek to examine the effect of CPT decision weight on Pratt's risk premium. Recall that the subjective risk premium may be defined as

$$
v\left(E_{y}^{s}[\Psi]-r_{S}^{\Psi}\right)=V_{y}[\Psi]
$$

They do not solve this equation for the risk premium explicitly, but rather show through the means of examples that the risk premium that attained may be either greater than or less than the standard Pratt risk premium in a model where no probability transformations take place. Specifically they look not at the risk premium itself, but at the market risk premium. That is, at the percentage premium of the expected value of the prospect to its subjective value. For the standard Pratt risk premium this is:

$$
m p=\frac{E_{y}[\Psi]}{E_{y}[\Psi]-r}-1
$$

where $r$ is the risk premium where no probability transformations are used in the valuation of the prospect. For a risk averse individual $r$ is positive, and $E_{y}[\Psi]-r$ is the certain amount for which this individual is indifferent to taking the gamble, which will thus be lower than the expected value. $m p$ is thus the percentage premium implied by this degree of risk aversion. With CPT probability distortions the market premium, $m p_{0}$, is

$$
m p_{0}=\frac{E_{y}[\Psi]}{E_{y}^{s}[\Psi]-r_{S}^{\Psi}}-1=\frac{E_{y}[\Psi]}{E_{y}[\Psi]-r^{\Psi}}-1
$$

Note that, although Levy and Levy use the subjective risk premium, the market risk premium remains the same regardless of whether the objective or subjective risk premium is used - the difference between the risk premia is exactly offset (definitionally) by the difference in the expected value used as the point of comparison.

Levy and Levy do not face the problem of probability "normalisations" in their examples 
here, as they use gains-only prospects in their analysis and their conclusion is that CPT decision weights may either increase or decrease the market risk premium, and thus the risk premium itself, We will now proceed in the next section to analyse these results in a generalised context, quantifying the effect on the risk premium of decision weights as well as reference dependent utility and loss aversion, all in the context of both the subjective and objective risk premia.

\subsubsection{Comprehensive Approximate Solutions with Non-linear Probability Weight- ings}

Objective Risk Premium In section 3 we provided a characterisation of the risk premium for small prospects in the case where there is no non-linear probability transformation. It still remains to ask what holds if the full CPT framework is applied, that is, in a world of non-linear probability weighting. We focus first on the objective risk premium. That is, the risk premium relative to the objective expected value of the distribution. This measure, $r^{\Psi}$, tells us the degree to which the decision maker actually displays risk aversion or risk seeking behaviour. This may differ from the subjective risk premium, $r_{S}^{\Psi}$ which indicates the degree to which the decision maker believes themselves to be risk averse or risk seeking (i.e., the risk premium is measured relative to the subjective expected value). In the case with CPT decision weights the approximate solutions from section 3 become more complex:

$$
\begin{aligned}
v\left(E_{y}\left[\Psi_{\varepsilon}\right]-r^{\Psi}\right) & =(1-p) E_{y}\left[v_{-}(\varepsilon) m_{-}(\varepsilon) \mid \varepsilon<0\right]+p E_{y}\left[v_{+}(\varepsilon) m_{+}(\varepsilon) \mid \varepsilon>0\right] \\
v\left(-r^{\Psi}\right) & =(1-p) E_{y}\left[v_{-}(\varepsilon) m_{-}(\varepsilon) \mid \varepsilon<0\right]+p E_{y}\left[v_{+}(\varepsilon) m_{+}(\varepsilon) \mid \varepsilon>0\right]
\end{aligned}
$$

recalling that

$$
m(x)=\left\{\begin{array}{c}
m_{+}(x)=w_{+}^{\prime}(1-F(x)) \text { for all } x \geq 0 \\
m_{-}(x)=w_{-}^{\prime}(F(x)) \text { for all } x<0
\end{array}\right.
$$

Considering only Case 2 where $r^{\Psi}>0$ (the analysis extends to the opposite case as before), the LHS approximation remains the same (as there is no uncertainty in the risk premium, and 
therefore no reweighting of probabilities). The RHS however, changes to give:

$$
\begin{aligned}
-\lambda v_{-}^{\prime}(0) r^{\Psi} \approx & \left(\begin{array}{c}
v_{-}^{\prime}(0) m_{-}(0) E_{y}[\varepsilon \mid \varepsilon<0] \\
+\frac{1}{2}\left(v_{-}^{\prime \prime}(0) m_{-}(0)+v_{-}^{\prime}(0) m_{-}^{\prime}(0)\right) E_{y}\left[\varepsilon^{2} \mid \varepsilon<0\right]
\end{array}\right) \lambda(1-p) \\
& +\left(\begin{array}{c}
v_{+}^{\prime}(0) m_{+}(0) E_{y}[\varepsilon \mid \varepsilon>0] \\
+\frac{1}{2}\left(v_{+}^{\prime \prime}(0) m_{+}(0)+v_{+}^{\prime}(0) m_{+}^{\prime}(0)\right) E_{y}\left[\varepsilon^{2} \mid \varepsilon>0\right]
\end{array}\right) p
\end{aligned}
$$

Using $E_{y}[\varepsilon \mid \varepsilon<0](1-p)=-E_{y}[\varepsilon \mid \varepsilon>0] p$ and $\lambda=\frac{v_{\uparrow}^{\prime}(0)}{v_{\downarrow}^{\prime}(0)}$ as before:

$$
\begin{aligned}
r^{\Psi} \approx & -\left(\begin{array}{c}
\left(m_{-}(0) E_{y}[\varepsilon \mid \varepsilon<0]+\frac{1}{2}\left(\frac{v_{-}^{\prime \prime}(0) m_{-}(0)}{v^{\prime}(0)}+m_{-}^{\prime}(0)\right) E_{y}\left[\varepsilon^{2} \mid \varepsilon<0\right]\right)(1-p) \\
+\left(m_{+}(0) E_{y}[\varepsilon \mid \varepsilon>0]+\frac{1}{2}\left(\frac{v_{+}^{\prime \prime}(0) m_{+}(0)}{v^{\prime}(0)}+m_{+}^{\prime}(0)\right) E_{y}\left[\varepsilon^{2} \mid \varepsilon>0\right]\right) \frac{1}{\lambda} p
\end{array}\right) \\
r^{\Psi} \approx & \left(\begin{array}{c}
\left.m_{-}(0)-\frac{1}{\lambda} m_{+}(0)\right) E_{y}[\varepsilon \mid \varepsilon>0] p \\
-
\end{array}\right. \\
& -\frac{1}{2}\left(\begin{array}{c}
(1-p)\left(\frac{v_{-}^{\prime \prime}(0) m_{-}(0)}{v^{\prime}(0)}+m_{-}^{\prime}(0)\right) E_{y}\left[\varepsilon^{2} \mid \varepsilon<0\right] \\
+\frac{1}{\lambda} p\left(\frac{v_{+}^{\prime \prime}(0) m_{+}(0)}{v^{\prime}(0)}+m_{+}^{\prime}(0)\right) E_{y}\left[\varepsilon^{2} \mid \varepsilon>0\right]
\end{array}\right)
\end{aligned}
$$

This leads as before to the sufficient conditions for risk aversion in CPT. These are given in the proposition below.

Proposition 8 Given a monotonically increasing utility function

$$
\begin{aligned}
& v(\varepsilon)=\left\{\begin{array}{c}
v_{+}(\varepsilon), \text { if } \varepsilon>0 \\
\lambda v_{-}(\varepsilon), \text { if } \varepsilon<0
\end{array}\right. \\
& v(0)=0
\end{aligned}
$$

and a non-linear decision weighting function $\pi(\varepsilon)$, applied separately to gains and losses

$$
m(\varepsilon)=\left\{\begin{array}{c}
m_{+}(\varepsilon)=w_{+}^{\prime}(1-F(\varepsilon)) \text { for all } \varepsilon \geq 0 \\
m_{-}(x)=w_{-}^{\prime}(F(\varepsilon)) \text { for all } \varepsilon<0
\end{array}\right.
$$


where $w^{+}$and $w^{-}$are two strictly increasing weighting functions $[0,1] \times \mathbb{R} \rightarrow[0,1]$ with $w^{+}(0)=$ $w^{-}(0)=0$ and $w^{+}(1)=w^{-}(1)=1$ and $F(\varepsilon)$ is cumulative probability distribution of $\varepsilon$, and where any continuous prospect is evaluated using continuous cumulative prospect theory with the reference point $y$. If we face a gamble with outcomes distributed according a random variable $\varepsilon$ representing deviations from the reference point $y$. The gamble has zero mean, $E_{y}\left[\Psi_{\varepsilon}\right]=E(\varepsilon)=$ 0 and infinitessimal variance, and

e)

$$
\lambda>\frac{m_{+}(0)}{m_{-}(0)}
$$

f)

$$
v_{-}^{\prime \prime}(0)<-\frac{m_{-}^{\prime}(0)}{m_{-}(0)} v^{\prime}(0)
$$

g)

$$
v_{+}^{\prime \prime}(0)<-\frac{m_{+}^{\prime}(0)}{m_{+}(0)} v^{\prime}(0)
$$

then the risk premium is positive, $r^{\Psi}>0$ (i.e., the individual is locally risk-averse for small changes in wealth)

As before these conditions can be seen as a condition on loss aversion, and a pair of conditions, analogous to the Pratt-Arrow curvature condition, on the curvature of the basic utility functions for gains and for losses. All of these conditions are changed by the introduction of rank-dependent probability weightings, and are related to the shape of the multiplier function, evaluated at the reference point. As would be expected, the conditions e), f) and g) collapse to those given in propositions 1 and 2 if the multiplier is everywhere equal to 1 . Thus, without any non-linear probability weightings, the sufficient conditions for a positive risk premium (and thus, overall risk aversion) are that there is loss aversion $(\lambda>1)$, and that the utility functions on basic utility are everywhere concave. With the introduction of the decision weighting function these conditions need to be adjusted to the forms given in the proposition above. 
Subjective Risk Premium The conditions given above ensure a positive objective risk premium where the risk aversion means the decision maker would rather take the objective expected value, than take the gamble. However, since the decision maker will only ever be aware of their subjective assessment of the probabilities in the gamble, it makes sense to also ask whether the decision maker would rather take the subjective expected value, $E_{y}^{s}[\Psi]$, rather than the gamble itself. A subjectively risk averse person would rather take her assessment of the expected value than the gamble itself (and $r_{S}^{\Psi}>0$ ). This person may, however, be simultaneously risk seeking in the objective sense $\left(r^{\Psi}<0\right)$.

As we show below, ensuring subjective risk aversion requires an additional condition to those which ensure objective risk aversion. In this case the subjective risk premium, $r_{S}^{\Psi}$, is defined as:

$$
v\left(E_{y}^{s}\left[\Psi_{\varepsilon}\right]-r_{S}^{\Psi}\right)=(1-p) E_{y}\left[v_{-}(\varepsilon) m_{-}(\varepsilon) \mid \varepsilon<0\right]+p E_{y}\left[v_{+}(\varepsilon) m_{+}(\varepsilon) \mid \varepsilon>0\right]
$$

In this case the approximation of the RHS remains exactly as above, but for the LHS we can no longer assume that $E_{y}^{s}\left[\Psi_{\varepsilon}\right]=0$ as this expectation may be distorted by subjective probability assessments. Thus we have as the LHS of (43):

$$
\begin{aligned}
L H S & =v\left(E_{y}^{s}\left[\Psi_{\varepsilon}\right]-r_{S}^{\Psi}\right) \\
& \approx \lambda v_{-}^{\prime}(0)\left(E_{y}^{s}\left[\Psi_{\varepsilon}\right]-r_{S}^{\Psi}\right)
\end{aligned}
$$

Using the same simplifications as before this leads to a subjective analogue to (44):

$$
\begin{aligned}
r_{S}^{\Psi} \approx & E_{y}^{s}\left[\Psi_{\varepsilon}\right]+\left(m_{-}(0)-\frac{1}{\lambda} m_{+}(0)\right) E_{y}[\varepsilon \mid \varepsilon>0] p \\
& -\frac{1}{2}\left(\begin{array}{c}
(1-p)\left(\frac{v_{-}^{\prime \prime}(0) m_{-}(0)}{v^{\prime}(0)}+m_{-}^{\prime}(0)\right) E_{y}\left[\varepsilon^{2} \mid \varepsilon<0\right] \\
+\frac{p}{\lambda}\left(\frac{v_{+}^{\prime \prime}(0) m_{+}(0)}{v^{\prime}(0)}+m_{+}^{\prime}(0)\right) E_{y}\left[\varepsilon^{2} \mid \varepsilon>0\right]
\end{array}\right)
\end{aligned}
$$

Apart from the first term on the right hand side, this has exactly the same form as (44). In order to guarantee subjective risk aversion for small prospects centred on the status quo, then, the addition condition required is that $E_{y}^{s}\left[\Psi_{\varepsilon}\right]>0$ (or equivalently $E_{y}^{s}\left[\Psi_{\varepsilon}\right]>E_{y}\left[\Psi_{\varepsilon}\right]$ ). Looked at another way this means that it is possible for decision makers to display objective risk aversion 
and have $r^{\Psi}<0$, and yet believe themselves to be risk neutral (or risk seeking) as $r_{S}^{\Psi}=0(>0)$. The opposite conclusion may also be true if $E_{y}^{s}\left[\Psi_{\varepsilon}\right]<E_{y}\left[\Psi_{\varepsilon}\right]$ : the decision maker may appear objectively risk seeking, but believe herself to be risk neutral or risk averse.

This relates to the subjectivity premium, $r_{\pi}^{\Psi}$, introduced in (14):

$$
r_{\pi}^{\Psi}=r^{\Psi}-r_{S}^{\Psi}=E_{y}[\Psi]-E_{y}^{s}[\Psi]=\int x f(x) d x-\int x m(x) f(x) d x
$$

The difference between the objective risk premium and the subjective risk premium depends only on the distortion of the expected value of the gamble that is induced by the probability distortions. For a small prospect centred on the reference point we have $E_{y}\left[\Psi_{\varepsilon}\right]=0$ and $E_{y}^{s}\left[\Psi_{\varepsilon}\right]=(1-p) E_{y}\left[m_{-}(\varepsilon) \mid \varepsilon<0\right]+p E_{y}\left[m_{+}(\varepsilon) \mid \varepsilon>0\right]$. Thus

$$
r_{\pi}^{\Psi}=-E_{y}^{s}[\Psi]=-\left((1-p) E_{y}\left[m_{-}(\varepsilon) \mid \varepsilon<0\right]+p E_{y}\left[m_{+}(\varepsilon) \mid \varepsilon>0\right]\right)
$$

\subsection{Discussion of Conditions of Risk Aversion at the Reference Point}

The conditions on loss aversion with subjective probability weightings may be further investigated by examining the conditions (45), (46) and (47) at the reference point, given what we know about the shape of decision weighting functions. If we take Prelec's (1998) one parameter form for the decision weighting function

$$
w_{+}(P)=w_{-}(P)=e^{-(-\ln P)^{\varphi}}
$$

then the multiplier functions at zero are given by

$$
\begin{aligned}
& m_{+}(0)=\frac{\varphi}{(1-F(0))}(-\ln (1-F(0)))^{\varphi-1} e^{-(-\ln (1-F(0)))^{\varphi}} \\
& m_{-}(0)=\frac{\varphi}{F(0)}(-\ln F(0))^{\varphi-1} e^{-(-\ln F(0))^{\varphi}}
\end{aligned}
$$

where $F(0)$ is the value of the cumulative probability distribution at the reference point.

Since the value of the cumulative distribution at $x=0(F(0))$ is equal to $1-p$, these can be 
simplified to

$$
\begin{aligned}
& m_{+}(0)=\frac{\varphi}{p}(-\ln (p))^{\varphi-1} e^{-(-\ln p)^{\varphi}} \\
& m_{-}(0)=\frac{\varphi}{1-p}(-\ln (1-p))^{\varphi-1} e^{-(-\ln (1-p))^{\varphi}}
\end{aligned}
$$

Figure 3 shows $m_{+}(0)$ as a function of $p$ for an inverse-S shaped Prelec decision weighting function, using the empirical estimate of $\mathrm{Wu}$ and Gonzalez (1996) of $\varphi=0.74$. The equivalent function for $m_{-}(0)$ is obtained by reflecting this function around $p=0.5$.

\section{Insert Figure 3}

\subsubsection{Loss Aversion Condition}

The ratio $\frac{m_{+}(0)}{m_{-}(0)}$ in the loss aversion condition is equal to

$$
\begin{aligned}
\frac{m_{+}(0)}{m_{-}(0)} & =\frac{(1-p)(-\ln p)^{\varphi-1} e^{-(-\ln p)^{\varphi}}}{p(-\ln (1-p))^{\varphi-1} e^{-(-\ln (1-p))^{\varphi}}} \\
& =\frac{1-p}{p}\left(\frac{\ln p}{\ln (1-p)}\right)^{\varphi-1} e^{-\left((-\ln (1-p))^{\varphi}+(-\ln p)^{\varphi}\right)}
\end{aligned}
$$

Loss aversion needs to be greater than this value to guarantee overall risk aversion. Using Prelec's function then, the condition on loss aversion is dependent only on the degree of non-linearity in the decision weighting $(\varphi)$, and the probability of getting a gain rather than a loss. Figure 4 below shows the dependency of the Loss Aversion sufficiency condition for the inverse-S shaped Prelec decision weighting function $(\varphi=0.74)$.

\section{Insert Figure 4}

At $p=50 \%$ the Loss Aversion parameter must be greater than 1 to guarantee a positive risk premium (in conjunction with the curvature conditions). As the probability of a gain increases from $50 \%$, the degree of loss aversion required to guarantee a positive risk premium increases to about $\lambda=1.3$, before dropping dramatically as the probability of a gain approaches unity. For $p<50 \%$ the condition may be satisfied even for loss loving individuals, except for distributions that approach loss-only distributions (i.e., with $p=0$ ).

It is important to bear in mind that the distribution retains an expected value of zero, 
regardless of the probability of a gain in this analysis. Therefore by varying $p$, we are implicitly skewing the distribution, although the variance remains negligibly small. By varying $p$ then, we are getting an idea of how the degree of local risk aversion (as measured by the risk premium) in the immediate vicinity of the reference point is affected by changing the proportions of gains vs. loss of the distribution whilst maintaining a constant mean.

The relationship away from the endpoints can be more clearly seen if we restrict the range of the graphic away from the extremes at $p=0$ and $p=1$, where the requirement diverge to positive and negative infinity respectively (Figure 5).

\section{Insert Figure 5}

\subsubsection{Concavity Conditions}

The other two conditions may be similarly examined. Figure 6 shows the relationship between the ratio $-\frac{m_{-}^{\prime}(0)}{m_{-}(0)}$ and the value of $p$.

\section{Insert Figure 6}

The equivalent relationship between $-\frac{m_{+}^{\prime}(0)}{m_{+}(0)}$ and $p$ looks very similar as it is the negative of the reflection of Figure 6 about the point $p=0.5$. Again, these relationships are clearer if we don't graph the function as $p$ approaches the extreme values of zero and one. This is shown for the ratio taken as the function approaches the reference point from the left hand side $\left(-\frac{m_{-}^{\prime}(0)}{m_{-}(0)}\right)$ in Figure 7 (i.e., a restricted view of Figure 6), and from the right hand side $\left(-\frac{m_{+}^{\prime}(0)}{m_{+}(0)}\right)$ in Figure 8 below.

\section{Insert Figures 7 and 8}

The concavity conditions required to ensure risk aversion are that the curvature of the loss utility function at the reference point must be less than the above functions multiplied by $v^{\prime}(0)$. This amounts to a simple condition of concavity for both gains and losses if $-\frac{m_{-}^{\prime}(0)}{m_{-}(0)}$ and $-\frac{m_{+}^{\prime}(0)}{m_{+}(0)}$ are both equal to zero. However, as can be seen from the Figures above, the functions are not simultaneously equal to zero for any value of $p$. Thus at least one of the two concavity conditions will be complicated by the addition of non-linear probability weightings.

To illustrate the effect of decision weights on the concavity condition for risk aversion in this context, consider the case when $p=0.5$. Here we have $-\frac{m_{-}^{\prime}(0)}{m_{-}(0)}>0$ and $-\frac{m_{+}^{\prime}(0)}{m_{+}(0)}<0$. Since $v^{\prime}(0)$ is greater than zero, this implies that the concavity condition on the gains utility function 
is strengthened: $v_{+}^{\prime \prime}(0)<-\frac{m_{+}^{\prime}(0)}{m_{+}(0)} v_{-}^{\prime}(0)<0$ so the utility function must be sufficiently concave. The condition on the loss utility function is weakened however, as the condition is given by $v_{-}^{\prime \prime}(0)<-\frac{m_{-}^{\prime}(0)}{m_{-}(0)} v_{-}^{\prime}(0)$, which is greater than zero. Thus, it is possible to guarantee a positive risk premium whilst having a loss utility function that is locally convex at the reference point, as long as it is not too convex. If the loss aversion condition is also met (which at $p=0.5$ requires that $\lambda>1$ ) then it is possible to have a positive risk premium whilst retaining the conditions that have been empirically observed, to wit, loss aversion, concavity for gains, and convexity for losses, with a utility function for losses that is more linear than that for gains.

In fact, this pattern can be accommodated for any value of $p$ below that where $-\frac{m_{-}^{\prime}(0)}{m_{-}(0)}=0$, or for extremely low values of $p$ where loss aversion exceeds the commonly observed value of about $\lambda=2.25$ (which only occurs below $p \approx 0.00001$ for $\varphi=0.74$ ). $-\frac{m_{-}^{\prime}(0)}{m_{-}(0)}=0$ occurs at $p=1 / e \approx 0.63$ regardless of the value of $\varphi$ (see Prelec 2000), so a positive risk premium can still be guaranteed with a convex utility function for losses as long as the probability of a gain is not too high, thus implying too long a tail for losses to ensure a zero expected value. If the probability of a gain is greater than $1 / e$ then a positive risk premium can only be guaranteed with a concave utility function for losses.

\subsection{Risk Aversion with Non-linear Probability Weights Away from the Reference Point}

The above analysis of risk aversion conditions with non-linear probability weightings has assumed throughout that we are examining prospects with small variance with the mean centered on the reference point. Often, if not usually, however, decision makers will be choosing between prospects that have positive or negative mean values. We now examine small variance prospects with non-zero means. In this case we will not have to employ both losses and gains in the solutions as the entire distribution is contained within an arbitrarily small range around the mean value $E_{y}[\varepsilon]$. The (objective) risk premium is defined here as:

$$
v\left(E_{y}[\varepsilon]-r^{\Psi}\right)=E_{y}[m(\varepsilon) v(\varepsilon)]
$$


where $m(\varepsilon)$ is either $m^{+}(\varepsilon)$ or $m^{-}(\varepsilon)$ as appropriate. Employing the now familiar approximations around the point $E_{y}[\varepsilon]$ yields

$$
L H S \approx v\left(E_{y}[\varepsilon]\right)-r^{\Psi} v^{\prime}\left(E_{y}[\varepsilon]\right)
$$

and

$$
\begin{aligned}
R H S & \approx E_{y}\left[\begin{array}{c}
v\left(E_{y}[\varepsilon]\right) m\left(E_{y}[\varepsilon]\right)+\left(\varepsilon-E_{y}[\varepsilon]\right)\left(v^{\prime}\left(E_{y}[\varepsilon]\right) m\left(E_{y}[\varepsilon]\right)+v\left(E_{y}[\varepsilon]\right) m^{\prime}\left(E_{y}[\varepsilon]\right)\right) \\
+\frac{1}{2}\left(\varepsilon-E_{y}[\varepsilon]\right)^{2}\left(\begin{array}{c}
v^{\prime \prime}\left(E_{y}[\varepsilon]\right) m\left(E_{y}[\varepsilon]\right)+2 v^{\prime}\left(E_{y}[\varepsilon]\right) m^{\prime}\left(E_{y}[\varepsilon]\right) \\
+v\left(E_{y}[\varepsilon]\right) m^{\prime \prime}\left(E_{y}[\varepsilon]\right)
\end{array}\right)
\end{array}\right] \\
\approx & v\left(E_{y}[\varepsilon]\right) m\left(E_{y}[\varepsilon]\right)+\frac{1}{2} \sigma^{2}\left(\begin{array}{c}
v^{\prime \prime}\left(E_{y}[\varepsilon]\right) m\left(E_{y}[\varepsilon]\right)+2 v^{\prime}\left(E_{y}[\varepsilon]\right) m^{\prime}\left(E_{y}[\varepsilon]\right) \\
+v\left(E_{y}[\varepsilon]\right) m^{\prime \prime}\left(E_{y}[\varepsilon]\right)
\end{array}\right)
\end{aligned}
$$

Setting these approximately equal to each other gives

$$
\begin{aligned}
v\left(E_{y}[\varepsilon]\right)-r^{\Psi} v^{\prime}\left(E_{y}[\varepsilon]\right) \approx & v\left(E_{y}[\varepsilon]\right) m\left(E_{y}[\varepsilon]\right)+\frac{1}{2} \sigma^{2}\left(\begin{array}{c}
v^{\prime \prime}\left(E_{y}[\varepsilon]\right) m\left(E_{y}[\varepsilon]\right)+2 v^{\prime}\left(E_{y}[\varepsilon]\right) m^{\prime}\left(E_{y}[\varepsilon]\right) \\
+v\left(E_{y}[\varepsilon]\right) m^{\prime \prime}\left(E_{y}[\varepsilon]\right)
\end{array}\right) \\
r^{\Psi} \approx & \frac{v\left(E_{y}[\varepsilon]\right)}{v^{\prime}\left(E_{y}[\varepsilon]\right)}\left(1-m\left(E_{y}[\varepsilon]\right)-\frac{\sigma^{2}}{2} m^{\prime \prime}\left(E_{y}[\varepsilon]\right)\right) \\
& -\sigma^{2} m^{\prime}\left(E_{y}[\varepsilon]\right)-\frac{\sigma^{2}}{2} \frac{v^{\prime \prime}\left(E_{y}[\varepsilon]\right)}{v^{\prime}\left(E_{y}[\varepsilon]\right)} m\left(E_{y}[\varepsilon]\right)
\end{aligned}
$$

The last term in this approximate solution is almost identical to the Pratt-Arrow risk premium, with the exception that it is multiplied by $m(E[\varepsilon])$. Since this function is always positive, the sign of this term is determined solely by the curvature of the utility function at $E[\varepsilon]$. From Figure 3 we see that $m(E[\varepsilon])<1$ (for an inverse-S shaped weighting function) unless the probability mass above and below the mean is highly asymmetrical. In these cases where $F(E[\varepsilon])$ is far from $0.5, m(E[\varepsilon])$ increases rapidly. For all distributions without extreme skew then, nonlinear probability weighting will dampen risk attitude expressed by this term towards risk neutrality.

The first term is the same sign as $v(E[\varepsilon])$ if $m(E[\varepsilon])+\frac{\sigma^{2}}{2} m^{\prime \prime}(E[\varepsilon])<1$. From the paragraph above we know the behaviour of $m(E[\varepsilon])$. Again examining Figure 3 we see that, for an inverse-S 
shaped weighting function we that $m^{\prime \prime}(E[\varepsilon])>0$ (i.e., it is everywhere convex) and relatively linear $\left(m^{\prime \prime}(E[\varepsilon])\right.$ close to 0$)$ as long as the distribution is not highly asymmetrical. Thus, for small variance distributions without extreme asymmetry, this term would increase risk aversion for gains, and decrease it for losses. This situation would reverse for highly asymmetric distributions (for which $m(E[\varepsilon])+\frac{\sigma^{2}}{2} m^{\prime \prime}(E[\varepsilon])>1$.

The middle term, $-\sigma^{2} m^{\prime}(E[\varepsilon])$, is negative if $m^{\prime}(E[\varepsilon])>0$, which occurs as the distribution becomes more negatively asymmetric for losses (that is as the probability mass below the mean, $F(E[\varepsilon])$, increases) and more positively asymmetric for gains $(F(1-E[\varepsilon])$ increases). For distributions without extreme symmetry $m^{\prime}(E[\varepsilon])$ is fairly close to zero and should not have a major impact on the risk premium.

The standard Pratt-Arrow risk premium $r^{\Psi} \approx-\frac{\sigma^{2}}{2} \frac{v^{\prime \prime}(E[\varepsilon])}{v^{\prime}(E[\varepsilon])}$ is a special case of equation (55) with no nonlinear probability transformations: $m(E[\varepsilon])=1$.

\section{Decision Making Example Employing Exact Solutions}

To complete this paper we provide an example of an exact solution for the risk premium using a particular distribution of outcomes, and show how this premium is influenced by the behavioural components of risk aversion, loss aversion, and decision weights. We choose for our distribution of outcomes a financial example, using excess returns for the US stock market over a twenty year period. This example is used primarily for the convenience and availability of long periods of financial data, but is intended to be purely illustrative.

Davies and Satchell (2003) show that the continuous CPT evaluation is explicitly solvable if one uses two power utility functions for gain and losses, a split Weibull distribution for returns (KST distribution (Knight, Satchell et al. 1995)), and Prelec's functional form for the probability weighting function (Prelec 1998). Köbberling and Wakker (2003) note that their loss aversion index $\lambda=\frac{v_{\uparrow}^{\prime}(0)}{v_{\downarrow}^{\prime}(0)}$ poses problems for the use of power utility functions. Following their discussion we employ exponential utility in our example.

Utility is thus given by: 


$$
\begin{aligned}
& v_{+}(x)=\frac{1-e^{-g x}}{g} \text { for } x \geq 0 \\
& v_{-}(x)=\frac{e^{l x}-1}{l} \text { for } x<0
\end{aligned}
$$

where $g$ and $l$ govern the curvature of the utility functions for gains and losses respectively and, where both are greater than zero we have the most common empirical situation of concavity for gains and convexity for losses.

The density function by:

$$
f(x)=\left\{\begin{array}{c}
p \frac{\alpha_{1}}{\beta_{1}^{\alpha_{1}}}(x)^{\alpha_{1}-1} e^{-\left(\frac{x}{\beta_{1}}\right)^{\alpha_{1}}} \text { for } x>0 \\
(1-p) \frac{\alpha_{2}}{\beta_{2}^{\alpha_{2}}}(-x)^{\alpha_{2}-1} e^{-\left(\frac{-x}{\beta_{2}}\right)^{\alpha_{2}}} \text { for } x \leq 0
\end{array}\right.
$$

where $\alpha_{1}$ and $\beta_{1}$ are parameters of the positive Weibull distribution, and $\alpha_{2}$ and $\beta_{2}$ are the Weibull parameters for losses. $p$ is the probability of being above the reference point. When fitted to the monthly excess returns of the S\&P 500 from October 1982 to September 2002 (using 3 month treasury bills as the risk free asset) using maximum likelihood estimation, the parameters are $\alpha_{1}=1.268, \beta_{1}=0.037, \alpha_{2}=1.087$, and $\beta_{2}=0.037 . p$ is equal to $60.83 \%$. The mean excess return is $E_{y}[\Psi]=0.671 \%$ per month (approx. $8.1 \%$ per year).

The cumulative distribution is given by:

$$
F(x)=\left\{\begin{array}{c}
(1-p)+p\left(1-e^{-\left(\frac{x}{\beta_{1}}\right)^{\alpha_{1}}}\right) \text { for } x>0 \\
(1-p)\left(-e^{-\left(\frac{-x}{\beta_{2}}\right)^{\alpha_{2}}}\right)
\end{array}\right.
$$

Prelec's weighting function gives:

$$
\begin{aligned}
& m_{+}(x)=\frac{\varphi}{(1-F(x))}(-\ln (1-F(x)))^{\varphi-1} e^{-(-\ln (1-F(x)))^{\varphi}} \\
& m_{-}(x)=\frac{\varphi}{F(x)}(-\ln F(x))^{\varphi-1} e^{-(-\ln F(x))^{\varphi}}
\end{aligned}
$$

with $1-F(x)$ as the decumulative distribution for gains, and $F(x)$ the cumulative distribution for losses. 
Let us also define for notational convenience:

$$
\begin{aligned}
u^{+} & \equiv p E_{y}\left[v_{+}(x) m_{+}(x) f(x) \mid x>0\right] \\
u^{-} & \equiv(1-p) E_{y}\left[\lambda v_{-}(x) m_{-}(x) f(x) \mid x<0\right]
\end{aligned}
$$

Using this notation $V_{y}[\Psi]$ becomes:

$$
\begin{aligned}
V_{y}[\Psi] & =\int_{-\infty}^{0} \lambda v_{-}(x) m_{-}(x) f(x) d x+\int_{0}^{\infty} v_{+}(x) m_{+}(x) f(x) d x \\
& =(1-p) \lambda E_{y}\left[v_{-}(x) m_{-}(x) f(x) \mid x<0\right]+p E_{y}\left[v_{+}(x) m_{+}(x) f(x) \mid x>0\right] \\
& =u^{+}+\lambda u^{-}
\end{aligned}
$$

These functional forms lead to the following equations which can be computed numerically (see Appendix for derivation):

$$
\begin{aligned}
& u^{+}=\frac{1}{g} \int_{(-\ln q)^{\varphi}}^{\infty} e^{-y}\left(1-e^{-g \beta_{1}\left(y^{\frac{1}{\varphi}}+\ln q\right)^{\frac{1}{\alpha_{1}}}}\right) d y \\
& u^{-}=\frac{1}{l} \int_{(-\ln (1-q))^{\varphi}}^{\infty} e^{-z}\left(e^{-l \beta_{2}\left(z^{\frac{1}{\varphi}}+\ln (1-q)\right)^{\frac{1}{\alpha_{2}}}}-1\right) d z
\end{aligned}
$$

Thus, for $E_{y}[\Psi]-r^{\Psi}>0$ :

$$
\begin{aligned}
r^{\Psi} & =E_{y}[\Psi]-v_{+}^{-1}\left(V_{y}[\Psi]\right) \\
& =E_{y}[\Psi]+\frac{\ln \left(1-\mu V_{y}[\Psi]\right)}{\mu} \\
& =E_{y}[\Psi]+\frac{\ln \left(1-\mu\left(u^{+}+\lambda u^{-}\right)\right)}{\mu}
\end{aligned}
$$


And for $E_{y}[\Psi]-r^{\Psi}<0$ :

$$
\begin{aligned}
r^{\Psi} & =E_{y}[\Psi]-v_{-}^{-1}\left(\frac{V_{y}[\Psi]}{\lambda}\right) \\
& =E_{y}[\Psi]-\frac{\ln \left(1+\nu\left(\frac{u^{+}+\lambda u^{-}}{\lambda}\right)\right)}{\nu}
\end{aligned}
$$

In what follows we explore these expressions and their sensitivity to the various parameters. Since $E_{y}[\Psi]=0.671 \%$, solutions with $r^{\Psi}<0.671 \%$ are taken from the first of these expressions (where the certain amount is evaluated as a gain), and solutions with $r^{\Psi}>0.671 \%$ will employ the second expression (66). Current theory and empirical data suggest that decision makers display concave basic utility for gains $(g>0)$, convex basic utility for losses $(l>0)$, loss aversion $(\lambda>1)$, and inverse-S shaped decision weighting functions $(0<\varphi<1)$. Specific estimates for the latter two suggest that values of $\lambda$ between 2 and 2.5 are appropriate (we shall employ 2.25 as our default) and $\varphi \simeq 0.74$ (Wu and Gonzalez 1996).

Using the default values for $\lambda$ and $\varphi$, Figure 9 shows the risk premium for concave gains and convex losses. For this distribution and a range for both $g$ and $l$ between 0 and 5 , the risk premium ranges between about $1.2 \%$ and $1.8 \%$. As expected it is increasing in the degree of concavity for gains, and deceasing in the amount of convexity for losses. These values are substantially higher than the expected excess returns of $E_{y}[\Psi]=0.671 \%$ - an investor with a risk premium in the indicated range would require a risk premium greater than the expected excess returns and would thus not purchase the index.

\section{Insert Figure 9}

Figure 10 illustrates the case where the decision maker displays convexity of basic utility for both gains and losses $(g<0, l>0)$. Unlike the situation with standard risk premia in EUT, these conditions do not automatically imply risk seeking behaviour for this returns distribution. In fact, the convexity has to be quite strong in both cases for risk seeking behaviour to result. This is because both loss aversion and non-linear decision weighting (with an inverse-S function) increase risk aversion. The kink in the surface occurs at $r^{\Psi}=0.671 \%$ and shows the effect of 
shifting from equation (66) to (65). For $0<r^{\Psi}<0.671 \%$ the risk premium is determined by (65) but is still positive, reflecting overall risk aversion.

\section{Insert Figure 10}

Figure 11 demonstrates the effects of Loss Aversion, and non-linear decision weights on the risk premium after setting $g=l=1$. We see that $r^{\Psi}$ is indeed increasing as loss aversion increases, and that it is decreasing in $\varphi$. No probability transformation is shown by the line at $\varphi=1$, with an inverse-S shaped weighting curve (as it most commonly observed) for $\varphi<1$, and an S-shaped curve for $\varphi>1$. Strong nonlinear decision weights can have a large effect on the risk premium.

\section{Insert Figure 11}

Lastly, Figure 12 examines the difference between the objective risk premium $r^{\Psi}$ and the subjective risk premium $r_{S}^{\Psi}$. The difference between them is equal to the difference between the expected value of the distribution $E_{y}[\Psi]$ and the subjective expected value $E_{y}^{s}[\Psi]$ as given by (14). We have (see Appendix for derivations):

$$
\begin{aligned}
E_{y}^{s}\left[\Psi_{\varepsilon}\right] & =(1-p) E_{y}\left[m_{-}(\varepsilon) \mid \varepsilon<0\right]+p E_{y}\left[m_{+}(\varepsilon) \mid \varepsilon>0\right] \\
& =\beta_{1} \int_{(-\ln q)^{\varphi}}^{\infty} e^{-y}\left(y^{\frac{1}{\varphi}}+\ln q\right)^{\frac{1}{\alpha_{1}}} d y-\beta_{2} \int_{(-\ln (1-q))^{\varphi}}^{\infty}\left(z^{\frac{1}{\varphi}}+\ln (1-q)\right)^{\frac{1}{\alpha_{2}}} e^{-z} d z(67)
\end{aligned}
$$

The subjectivity premium $r_{\pi}^{\Psi}=E_{y}[\Psi]-E_{y}^{s}[\Psi]$ is subtracted from $r^{\Psi}$ to give the subjective (Pratt-Arrow) risk premium $r_{S}^{\Psi}$. This subjectivity premium is positive for $\varphi<1$, zero for no probability transformations $(\varphi=0)$, and negative for S-shaped utility curves $(\varphi>1)$. Thus for US equity returns, non-linear decision weights with in inverse-S shaped weighting function causes the subjective risk premium to be less than the objective premium: $r_{S}^{\Psi}<r^{\Psi}$. In other words, individuals who distort probability in this way will believe themselves to be less risk averse than they actually are. However, we can also see from the chart that it is possible for the two risk premia to have different signs. For values of $\varphi$ in the region of approximately 1.1 to 1.4 , the decision makers believe themselves to be risk averse, whereas from an objective point of view the are actually behaving as risk seekers. These results show the considerable effect that this new concept of the subjectivity premium can have on risk attitudes. 


\section{Insert Figure 12}

\section{Concluding Comments}

In this paper we have shown that the standard characterisation of risk attitude as being driven by the curvature of the utility function glosses many nuances of the potential behavioural origins of risk aversion. When we set aside Expected Utility Theory in favour of a more behaviourally sophisticated model of decision making, such as the reference and rank dependent models as exemplified by $\mathrm{CPT}$, we see that risk attitude may arise in complex ways from the interaction of a number of distinct psychological or psychophysical reactions. In particular, whilst utility curvature still plays an important role in risk attitude, it now becomes important to distinguish between the gain and loss domains when considering the effect of curvature. The most common empirical result of concave reactions to gains and convex reactions to losses (which may be theoretically defended by an appeal to diminishing sensitivity away from the reference point) means that curvature for gains and for losses have quite distinct, and opposite, roles in determining overall risk aversion. In addition, risk attitude is affected by two other behavioural components: the tendency to treat losses with greater consideration than gains, and the tendency to distort probabilities in a rank-dependent fashion. Both of these concepts have effects on the risk premium that may be entirely separated from the traditional effects of utility curvature.

Our paper has provided both general and approximate local solutions for the risk premium that show the effects of each of the behavioural components individually. In addition we have provided the conditions on the behavioural components that are required to ensure local risk aversion. These conditions are the behavioural analogues of the traditional Pratt-Arrow condition of concave utility. The more sophisticated behavioural models allow us to decompose risk measurement into more meaningful effects than has hitherto been possible and also to be more precise in our language and analysis of what the effects of a given change in behaviour will be. 


\section{Appendix}

\subsection{Derivation of Exact Solutions}

Where excess returns are distributed according to

$$
f(x)=\left\{\begin{array}{l}
p f^{+}(x) \text { for } x \geq 0 \\
(1-p) f^{-}(-x) \text { for } x<0
\end{array}\right.
$$

for which the cumulative distribution is

$$
F(x)=\left\{\begin{array}{ccc}
(1-p)\left(1-F^{-}(-x)\right) & \text { if } & x<0 \\
(1-p)+p F^{+}(x) & \text { if } & x \geq 0
\end{array}\right.
$$

And the outcomes are distributed according to a Weibull distribution we have:

$$
\begin{gathered}
f^{+}\left(x ; \alpha_{1}, \beta_{1}\right)=\frac{\alpha_{1}}{\beta_{1}^{\alpha_{1}}} x^{\alpha_{1}-1} e^{-\left(\frac{x}{\beta_{1}}\right)^{\alpha_{1}}}, 0<x<\infty \\
F^{+}\left(x ; \alpha_{1}, \beta_{1}\right)=1-e^{-\left(\frac{x}{\beta_{1}}\right)^{\alpha_{1}}}, 0<x<\infty
\end{gathered}
$$

\subsubsection{Gains}

The imputed gains distribution $\pi^{+}(x)$ is arrived at by multiplying the objective distribution $f^{+}\left(x ; \alpha_{1}, \beta_{1}\right)$ by the multiplier $m^{+}(x)=w^{\prime+}\left(p\left(1-F^{+}(x)\right)\right)$ which, when using Prelec's weighting function is 


$$
\begin{aligned}
& m^{+}(x)=\frac{\varphi}{p\left(1-F^{+}(x)\right)}\left(-\ln p\left(1-F^{+}(x)\right)\right)^{\varphi-1} e^{-\left(-\ln p\left(1-F^{+}(x)\right)\right)^{\varphi}} \\
& =\frac{\varphi}{p e^{-\left(\frac{x}{\beta_{1}}\right)^{\alpha_{1}}}}\left(-\ln p e^{-\left(\frac{x}{\beta_{1}}\right)^{\alpha_{1}}}\right)^{\varphi-1} e^{-\left(-\ln p e^{-\left(\frac{x}{\beta_{1}}\right)^{\alpha_{1}}}\right)^{\varphi}} \\
& =\frac{\varphi}{p} e^{\left(\frac{x}{\beta_{1}}\right)^{\alpha_{1}}}\left(\left(\frac{x}{\beta_{1}}\right)^{\alpha_{1}}-\ln p\right)^{\varphi-1} e^{-\left(\left(\frac{x}{\beta_{1}}\right)^{\alpha_{1}}-\ln p\right)^{\varphi}} \\
& =\frac{\varphi}{p}\left(\left(\frac{x}{\beta_{1}}\right)^{\alpha_{1}}-\ln p\right)^{\varphi-1} e^{\left(\frac{x}{\beta_{1}}\right)^{\alpha_{1}}-\left(\left(\frac{x}{\beta_{1}}\right)^{\alpha_{1}}-\ln p\right)^{\varphi}}
\end{aligned}
$$

Therefore the imputed distribution is:

$$
\begin{aligned}
\pi^{+}(x) & =m^{+}(x) p f^{+}\left(x ; \alpha_{1}, \beta_{1}\right) \\
& =\frac{\varphi}{p}\left(\left(\frac{x}{\beta_{1}}\right)^{\alpha_{1}}-\ln p\right)^{\varphi-1} e^{\left(\frac{x}{\beta_{1}}\right)^{\alpha_{1}}-\left(\left(\frac{x}{\beta_{1}}\right)^{\alpha_{1}}-\ln p\right)^{\varphi} \frac{p \alpha_{1}}{\beta_{1}^{\alpha_{1}}} x^{\alpha_{1}-1} e^{-\left(\frac{x}{\beta_{1}}\right)^{\alpha_{1}}}} \\
& =\frac{\varphi \alpha_{1}}{\beta_{1}^{\alpha_{1}}} x^{\alpha_{1}-1}\left(\left(\frac{x}{\beta_{1}}\right)^{\alpha_{1}}-\ln p\right)^{\varphi-1} e^{-\left(\left(\frac{x}{\beta_{1}}\right)^{\alpha_{1}}-\ln p\right)^{\varphi}}
\end{aligned}
$$

\subsubsection{Losses}

For losses the objective pdf is:

$$
f^{-}\left(-x ; \alpha_{2}, \beta_{2}\right)=\frac{\alpha_{2}}{\beta_{2}^{\alpha 2}}(-x)^{\alpha_{2}-1} e^{-\left(\frac{-x}{\beta_{2}}\right)^{\alpha_{2}}},-\infty<x<0
$$

and the associated cdf is:

$$
F^{-}\left(-x ; \alpha_{2}, \beta_{2}\right)=1-e^{-\left(\frac{-x}{\beta_{2}}\right)^{\alpha_{2}}},-\infty<x<0
$$

The multiplier function is: 


$$
\begin{aligned}
& m^{-}(x)=w^{-}\left((1-p)\left(1-F^{-}(-x)\right)\right) \\
& =\frac{\varphi}{\left((1-p)\left(1-F^{-}(-x)\right)\right)}\left(-\ln (1-p)\left(1-F^{-}(-x)\right)\right)^{\varphi-1} e^{-\left(-\ln (1-p)\left(1-F^{-}(-x)\right)\right)^{\varphi}} \\
& =\frac{\varphi}{\left((1-p) e^{-\left(\frac{-x}{\beta_{2}}\right)^{\alpha_{2}}}\right)}\left(-\ln (1-p) e^{-\left(\frac{-x}{\beta_{2}}\right)^{\alpha_{2}}}\right)^{\varphi-1} e^{-\left(-\ln (1-p) e^{-\left(\frac{-x}{\beta_{2}}\right)^{\alpha_{2}}}\right)^{\varphi}} \\
& =\frac{\varphi}{(1-p)}\left(\left(\frac{-x}{\beta_{2}}\right)^{\alpha_{2}}-\ln (1-p)\right)^{\varphi-1} e^{\left(\frac{-x}{\beta_{2}}\right)^{\alpha_{2}}-\left(\left(\frac{-x}{\beta_{2}}\right)^{\alpha_{2}}-\ln (1-p)\right)^{\varphi}}
\end{aligned}
$$

Combining these as before to form the imputed distribution $\pi^{-}(x)$ :

$$
\begin{aligned}
\pi^{-}(x) & =m^{-}(x)(1-p) f^{-}\left(-x ; \alpha_{2}, \beta_{2}\right) \\
& =\frac{\varphi}{(1-p)}\left(\left(\frac{-x}{\beta_{2}}\right)^{\alpha_{2}}-\ln (1-p)\right)^{\varphi-1} e^{\left(\frac{-x}{\beta_{2}}\right)^{\alpha_{2}}-\left(\left(\frac{-x}{\beta_{2}}\right)^{\alpha_{2}}-\ln (1-p)\right)^{\varphi}} \frac{(1-p) \alpha_{2}}{\beta_{2}^{\alpha 2}}(-x)^{\alpha_{2}-1} e^{-\left(\frac{-x}{\beta_{2}}\right)^{\alpha_{2}}} \\
& =\frac{\varphi \alpha_{2}}{\beta_{2}^{\alpha 2}}(-x)^{\alpha_{2}-1}\left(\left(\frac{-x}{\beta_{2}}\right)^{\alpha_{2}}-\ln (1-p)\right)^{\varphi-1} e^{-\left(\left(\frac{-x}{\beta_{2}}\right)^{\alpha_{2}}-\ln (1-p)\right)^{\varphi}}
\end{aligned}
$$

\subsection{Derivation of $u^{+}$for Mixed Functions}

$u^{+}$is

$$
\begin{aligned}
u^{+} & =p E_{B}\left(v_{+}(x) \mid x>0\right) \\
& =\int_{0}^{\infty} v_{+}(x) m^{+}(x) p f^{+}(x) d x \\
& =\int_{0}^{\infty} v_{+}(x) \pi^{+}(x) d x
\end{aligned}
$$

where $E_{B}$ is the subjective expected value including probability weights, loss aversion, and utility curvature. 
Combining the Weibull-KST distribution and the multiplication function to form $u^{+}$:

$$
\begin{aligned}
u^{+} & =\int_{0}^{\infty} \frac{1-e^{-g x}}{g} \pi^{+}(x) d x \\
& =\int_{0}^{\infty} \frac{1-e^{-g x}}{g} \frac{\varphi \alpha_{1}}{\beta_{1}^{\alpha_{1}}} x^{\alpha_{1}-1}\left(\left(\frac{x}{\beta_{1}}\right)^{\alpha_{1}}-\ln p\right)^{\varphi-1} e^{-\left(\left(\frac{x}{\beta_{1}}\right)^{\alpha_{1}}-\ln p\right)^{\varphi}} d x \\
& =\frac{\varphi \alpha_{1}}{g \beta_{1}^{\alpha_{1}}} \int_{0}^{\infty}\left(1-e^{-g x}\right) x^{\alpha_{1}-1}\left(\left(\frac{x}{\beta_{1}}\right)^{\alpha_{1}}-\ln p\right)^{\varphi-1} e^{-\left(\left(\frac{x}{\beta_{1}}\right)^{\alpha_{1}}-\ln p\right)^{\varphi}} d x
\end{aligned}
$$

Let $y=\left(\left(\frac{x}{\beta_{1}}\right)^{\alpha_{1}}-\ln p\right)^{\varphi}$,

then $x=\beta_{1}\left(y^{\frac{1}{\varphi}}+\ln p\right)^{\frac{1}{\alpha_{1}}}$

and $d x=\frac{\beta_{1}}{\varphi \alpha_{1}}\left(y^{\frac{1}{\varphi}}+\ln p\right)^{\frac{1}{\alpha_{1}}-1} y^{\frac{1}{\varphi}-1} d y$

Substituting,

$$
\begin{aligned}
u^{+} & =\frac{\varphi \alpha_{1}}{g \beta_{1}^{\alpha_{1}}} \int_{(-\ln p)^{\varphi}}^{\infty}\left(1-e^{-g \beta_{1}\left(y^{\frac{1}{\varphi}}+\ln p\right)^{\frac{1}{\alpha_{1}}}}\right)\left(\beta_{1}\left(y^{\frac{1}{\varphi}}+\ln p\right)^{\frac{1}{\alpha_{1}}}\right)^{\alpha_{1}-1} y^{1-\frac{1}{\varphi}} e^{-y} \frac{\beta_{1}}{\varphi \alpha_{1}}\left(y^{\frac{1}{\varphi}}+\ln p\right)^{\frac{1}{\alpha_{1}}-1} y^{\frac{1}{\varphi}-1} d y \\
& =\frac{1}{g} \int_{(-\ln p)^{\varphi}}^{\infty} e^{-y}\left(1-e^{-g \beta_{1}\left(y^{\frac{1}{\varphi}}+\ln p\right)^{\frac{1}{\alpha_{1}}}}\right) d y
\end{aligned}
$$

\subsection{Derivation of $u^{-}$for Mixed Functions}

To solve over the loss domain,

$$
\begin{aligned}
u^{-} & =\int_{-\infty}^{0} v_{-}(x)(1-p) f^{-}(-x) m^{-}(x) d x \\
& =\int_{-\infty}^{0} \frac{e^{l x}-1}{l} \pi^{-}(x) d x \\
& =\frac{\varphi \alpha_{2}}{l \beta_{2}^{\alpha 2}} \int_{-\infty}^{0}\left(e^{l x}-1\right)(-x)^{\alpha_{2}-1}\left(\left(\frac{-x}{\beta_{2}}\right)^{\alpha_{2}}-\ln (1-p)\right)^{\varphi-1} e^{-\left(\left(\frac{-x}{\beta_{2}}\right)^{\alpha_{2}}-\ln (1-p)\right)^{\varphi}} d x \\
\text { Let } z & =\left(\left(\frac{-x}{\beta_{2}}\right)^{\alpha_{2}}-\ln (1-p)\right)^{\varphi},
\end{aligned}
$$


then $x=-\beta_{2}\left(z^{\frac{1}{\varphi}}+\ln (1-p)\right)^{\frac{1}{\alpha_{2}}}$

and $d x=-\frac{\beta_{2}}{\varphi \alpha_{2}}\left(z^{\frac{1}{\varphi}}+\ln (1-p)\right)^{\frac{1}{\alpha_{2}}-1} z^{\frac{1}{\varphi}-1} d z$

The value of $u^{-}$follows as before:

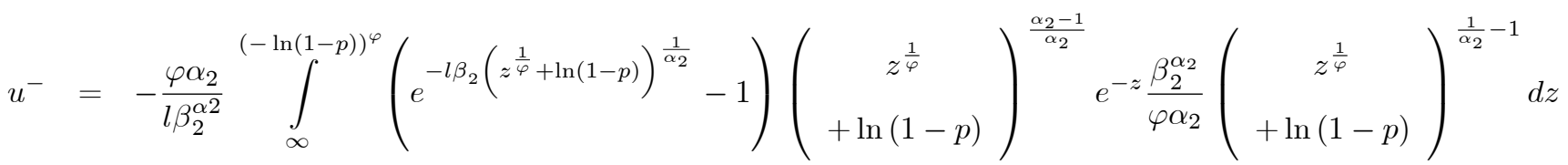

$$
\begin{aligned}
& =-\frac{1}{l} \int_{\infty}^{(-\ln (1-p))^{\varphi}} e^{-z}\left(e^{-l \beta_{2}\left(z^{\frac{1}{\varphi}}+\ln (1-p)\right)^{\frac{1}{\alpha_{2}}}}-1\right) d z \\
& =\frac{1}{l} \int_{(-\ln (1-p))^{\varphi}}^{\infty} e^{-z}\left(e^{-l \beta_{2}\left(z^{\frac{1}{\varphi}}+\ln (1-p)\right)^{\frac{1}{\alpha_{2}}}}-1\right) d z
\end{aligned}
$$

5.4 Subjective Expected Value

$$
\begin{aligned}
p E_{y}\left[m_{+}(\varepsilon) \mid \varepsilon>0\right] & =\int_{0}^{\infty} x f^{+}(x) p m^{+}(x) d x \\
& =\int_{0}^{\infty} x \pi^{+}(x) d x \\
& =\int_{0}^{\infty} \frac{\varphi \alpha_{1}}{\beta_{1}^{\alpha_{1}}} x^{\alpha_{1}}\left(\left(\frac{x}{\beta_{1}}\right)^{\alpha_{1}}-\ln p\right)^{\varphi-1} e^{-\left(\left(\frac{x}{\beta_{1}}\right)^{\alpha_{1}}-\ln p\right)^{\varphi}} d x \\
& =\frac{\varphi \alpha_{1}}{\beta_{1}^{\alpha_{1}}} \int_{0}^{\infty} x^{\alpha_{1}}\left(\left(\frac{x}{\beta_{1}}\right)^{\alpha_{1}}-\ln p\right)^{\varphi-1} e^{-\left(\left(\frac{x}{\beta_{1}}\right)^{\alpha_{1}}-\ln p\right)^{\varphi}} d x
\end{aligned}
$$

Let $y=\left(\left(\frac{x}{\beta_{1}}\right)^{\alpha_{1}}-\ln p\right)^{\varphi}$,

then $x=\beta_{1}\left(y^{\frac{1}{\varphi}}+\ln p\right)^{\frac{1}{\alpha_{1}}}$

and $d x=\frac{\beta_{1}}{\varphi \alpha_{1}}\left(y^{\frac{1}{\varphi}}+\ln p\right)^{\frac{1}{\alpha_{1}}-1} y^{\frac{1}{\varphi}-1} d y$

48 
Substituting,

$$
\begin{aligned}
p E_{y}\left[m_{+}(\varepsilon) \mid \varepsilon>0\right] & =\frac{\varphi \alpha_{1}}{\beta_{1}^{\alpha_{1}}} \int_{(-\ln p)^{\varphi}}^{\infty}\left(\beta_{1}\left(y^{\frac{1}{\varphi}}+\ln p\right)^{\frac{1}{\alpha_{1}}}\right)^{\alpha_{1}} y^{1-\frac{1}{\varphi}} e^{-y} \frac{\beta_{1}}{\varphi \alpha_{1}}\left(y^{\frac{1}{\varphi}}+\ln p\right)^{\frac{1}{\alpha_{1}}-1} y^{\frac{1}{\varphi}-1} d y \\
& =\beta_{1} \int_{(-\ln p)^{\varphi}}^{\infty} e^{-y}\left(y^{\frac{1}{\varphi}}+\ln p\right)^{\frac{1}{\alpha_{1}}} d y
\end{aligned}
$$

Similarly, for losses:

$$
\begin{aligned}
(1-p) E_{y}\left[m_{-}(\varepsilon) \mid \varepsilon<0\right] & =\int_{-\infty}^{0} \frac{\varphi \alpha_{2}}{\beta_{2}^{\alpha 2}}(-x)^{\alpha_{2}-1} x\left(\left(\frac{-x}{\beta_{2}}\right)^{\alpha_{2}}-\ln (1-p)\right)^{\varphi-1} e^{-\left(\left(\frac{-x}{\beta_{2}}\right)^{\alpha_{2}}-\ln (1-p)\right)^{\varphi}} d x \\
& =-\frac{\varphi \alpha_{2}}{\beta_{2}^{\alpha 2}} \int_{-\infty}^{0}(-x)^{\alpha_{2}}\left(\left(\frac{-x}{\beta_{2}}\right)^{\alpha_{2}}-\ln (1-p)\right)^{\varphi-1} e^{-\left(\left(\frac{-x}{\beta_{2}}\right)^{\alpha_{2}}-\ln (1-p)\right)^{\varphi}} d x
\end{aligned}
$$

Let $z=\left(\left(\frac{-x}{\beta_{2}}\right)^{\alpha_{2}}-\ln (1-p)\right)^{\varphi}$,

then $-x=\beta_{2}\left(z^{\frac{1}{\varphi}}+\ln (1-p)\right)^{\frac{1}{\alpha_{2}}}$

and $d x=-\frac{\beta_{2}}{\varphi \alpha_{2}}\left(z^{\frac{1}{\varphi}}+\ln (1-p)\right)^{\frac{1}{\alpha_{2}}-1} z^{\frac{1}{\varphi}-1} d z$

$$
\begin{aligned}
(1-p) E_{y}\left[m_{-}(\varepsilon) \mid \varepsilon<0\right] & =\frac{\varphi \alpha_{2}}{\beta_{2}^{\alpha 2}} \int_{\infty}^{(-\ln (1-p))^{\varphi}}\left(z^{\frac{1}{\varphi}}+\ln (1-p)\right) e^{-z} \frac{\beta_{2}^{\alpha_{2}+1}}{\varphi \alpha_{2}}\left(z^{\frac{1}{\varphi}}+\ln (1-p)\right)^{\frac{1}{\alpha_{2}}-1} d z \\
& =\beta_{2} \int_{\infty}^{(-\ln (1-p))^{\varphi}} e^{-z}\left(z^{\frac{1}{\varphi}}+\ln (1-p)\right)^{\frac{1}{\alpha_{2}}} d z \\
& =-\beta_{2} \int_{(-\ln (1-p))^{\varphi}}^{\infty}\left(z^{\frac{1}{\varphi}}+\ln (1-p)\right)^{\frac{1}{\alpha_{2}}} e^{-z} d z
\end{aligned}
$$




\section{Notes}

${ }^{1}$ This condition is implied by symmetry about 0 , but does not imply it.

${ }^{2}$ We have made some changes to their notation to fit with that of the present article. 


\section{Bibliography}

1. Abdellaoui, Mohammed. (2000). "Parameter-Free Elicitation of Utility of Probability Weighting Functions", Management Science 46, 1497-1512.

2. Arrow, Kenneth. (1965). Aspects of the Theory of Risk Bearing. Helsinki: Yrjo Jahnssonin Saatio.

3. Bernartzi, Shlomo and Richard H. Thaler. (1995). "Myopic Loss Aversion and the Equity Premium Puzzle", Quarterly Journal of Economics 110, 73-92.

4. Davies, Greg B. (2004). "Pure Risk: Reintroducing Risk into Rational Choice", Working Paper, Faculty of Economics and Politics, University of Cambridge.

5. Davies, Greg B. and Stephen E. Satchell. (2003). "Cumulative Prospect Theory and Individual Asset Allocation", Working Paper, Faculty of Economics and Politics, University of Cambridge.

6. Gonzalez, Richard and George Wu. (1999). "On the Shape of the Probability Weighting Function", Cognitive Psychology 38, 129-166.

7. Hilton, Ronald W. (1988). "Risk Attitude under Two Alternative Theories of Choice under Risk", Journal of Economic Behavior and Organization 9, 119-136.

8. Kahneman, Daniel and Amos Tversky. (1979). "Prospect Theory: An Analysis of Decision under Risk", Econometrica 47, 263-291.

9. Knight, J. L., S. E. Satchell, and K.C. Tran. (1995). "Statistical Modelling of Asymmetric Risk in Asset Returns", Applied Mathematical Finance 2, 155-172.

10. Köbberling, Veronika and Peter P. Wakker. (2003). "An Index of Loss Aversion", Working Paper, Department of Quantitative Economics, University of Maastricht.

11. Levy, Haim and Moshe Levy. (2002). "Arrow-Pratt Risk Aversion, Risk Premium and Decision Weights", Journal of Risk and Uncertainty 25, 265-290.

12. Mehra, R. and Edward C. Prescott. (1985). "The Equity Premium Puzzle: A Solution?" Journal of Monetary Economics XV, 145-161. 
13. Pratt, J. W. (1964). "Risk Aversion in the Small and in the Large", Econometrica 32, 122-136.

14. Prelec, Drazen. (1998). "The Probability Weighting Function", Econometrica 60, 497-528.

15. Prelec, Drazen. (2000). "Compound Invariant Weighting Functions in Prospect Theory". In Daniel Kahneman and Amos Tversky (eds.), Choices, Values, and Frames. Cambridge: Cambridge University Press.

16. Quiggin, J. (1982). "A Theory of Anticipated Utility", Journal of Economic Behavior and Organization 3, 323-343.

17. Schmidt, Ulrich. (2003). "Reference Dependence in Cumulative Prospect Theory", Journal of Mathematical Psychology 47, 122-131.

18. Sugden, Robert. (2003). "Reference-Dependent Subjective Expected Utility", Journal of Economic Theory 111, 172-191.

19. Tversky, Amos and Daniel Kahneman. (1992). "Advances in Prospect Theory: Cumulative Representation of Uncertainty", Journal of Risk and Uncertainty 5, 297-323.

20. Wu, George and Richard Gonzalez. (1996). "Curvature of the Probability Weighting Function", Management Science 42, 1676-1690.

21. Wu, George and Richard Gonzalez. (1999). "Nonlinear Decision Weights in Choice under Uncertainty", Management Science 45, 74-85. 


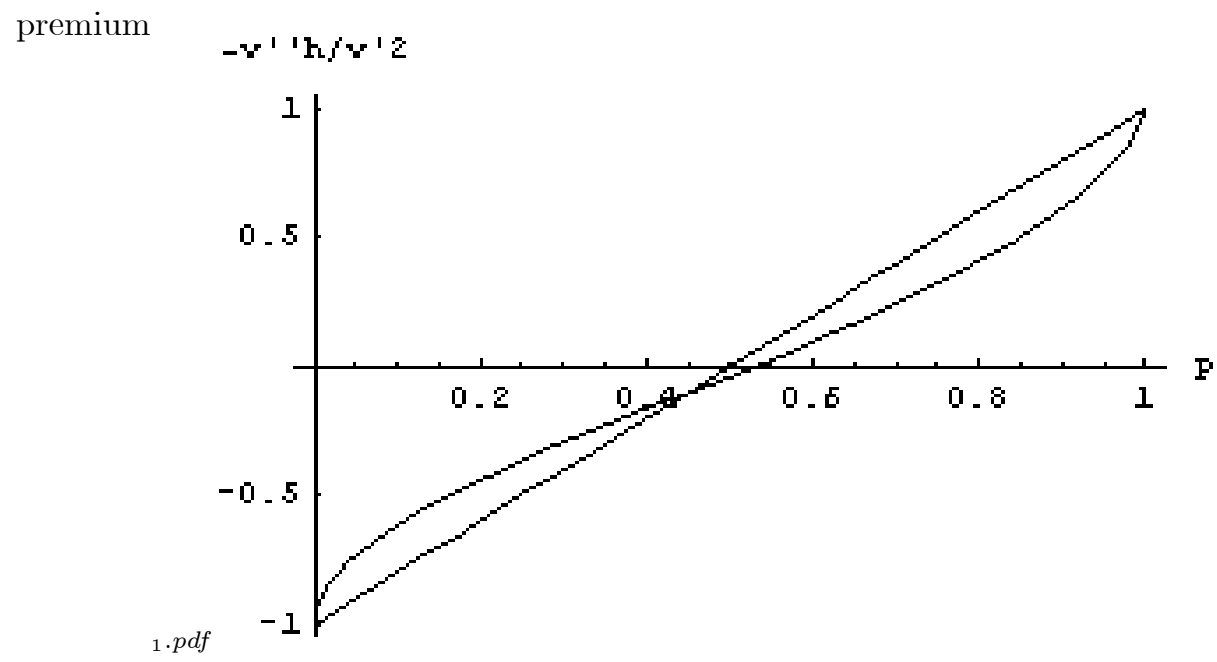

Figure 1: Arrow's risk premium without probability distortion, $p$, and with probability distortion, $p_{0}$, plotted against $-\frac{v^{\prime \prime}(0)}{v^{\prime}(0)} \frac{h}{2}$ using Tversky and Kahneman's weighting function with $\delta=0.69$, and $\gamma=0.61$. If $p_{0}>p$ for a given value of $-\frac{v^{\prime \prime}(0)}{v^{\prime}(0)} \frac{h}{2}$ indicates that probability distortion causes the decision maker to be more risk averse.

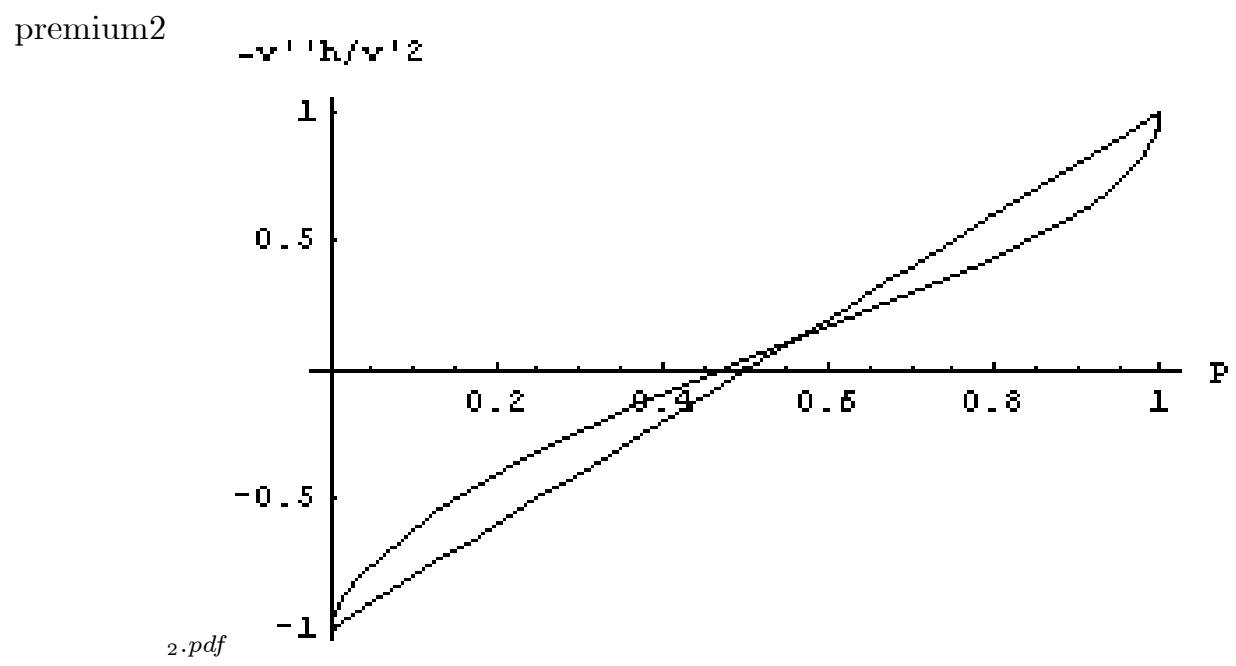

Figure 2: Arrow's risk premium without probability distortion, $p$, and with probability distortion, $p_{0}$, plotted against $-\frac{v^{\prime \prime}(0)}{v^{\prime}(0)} \frac{h}{2}$ using Tversky and Kahneman's weighting function with $\delta=0.61$, and $\gamma=0.69$. If $p_{0}>p$ for a given value of $-\frac{v^{\prime \prime}(0)}{v^{\prime}(0)} \frac{h}{2}$ indicates that probability distortion causes the decision maker to be more risk averse. In this case, the change in the parameters causes Levy and Levy's conclusion that probability distortion always increases risk aversion to no longer be valid. 


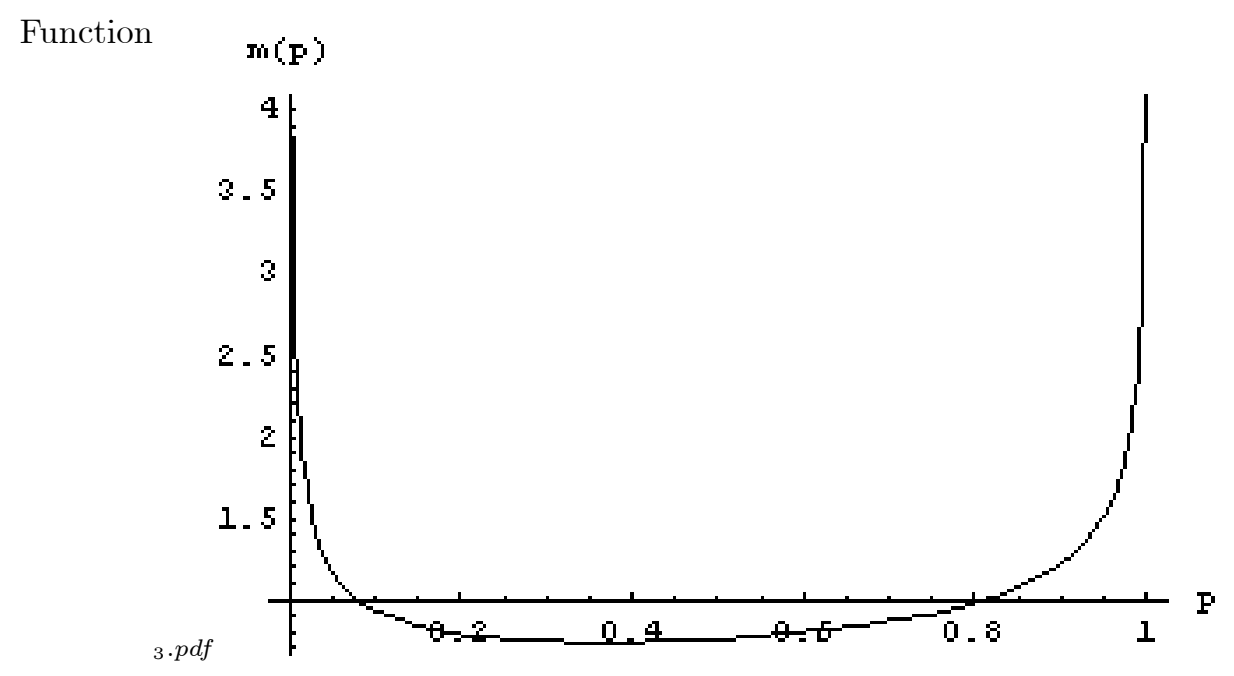

Figure 3: Gains Multiplier Function at reference point, $m_{+}(0)$

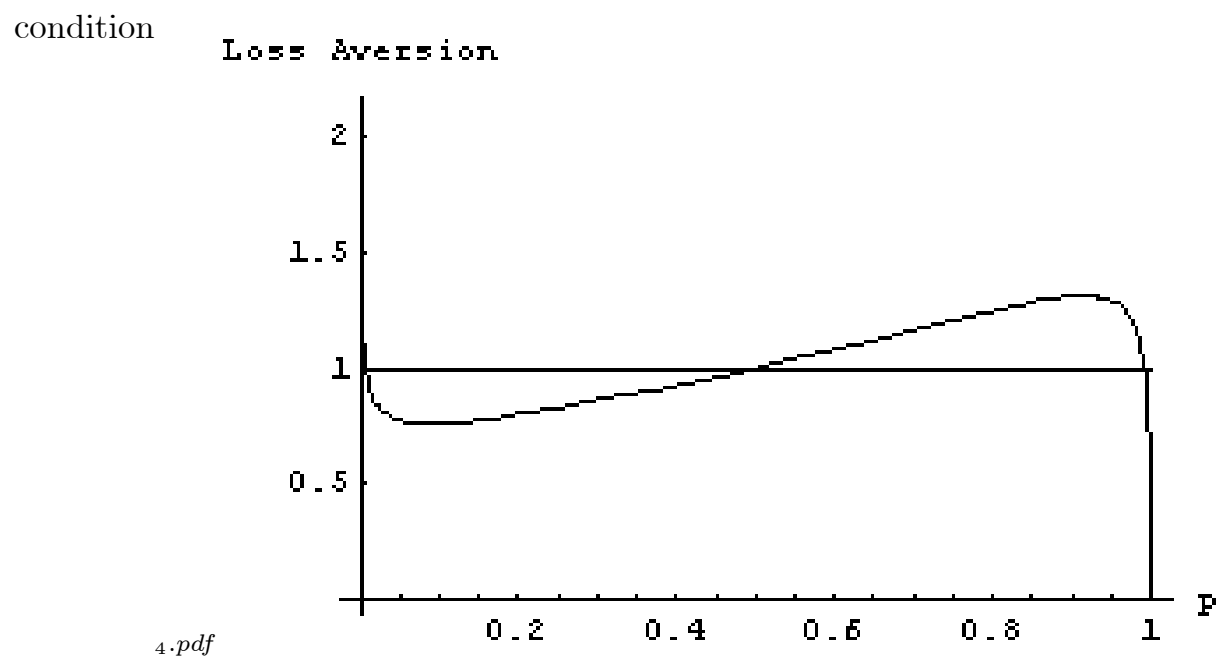

Figure 4: Loss Aversion sufficiency condition for positive risk premium as a function of the probability of a gain $(p)$ with the inverse-S shaped decision weighting function $(\varphi=0.74)$ 
condition internal

\section{Loss Aversion}

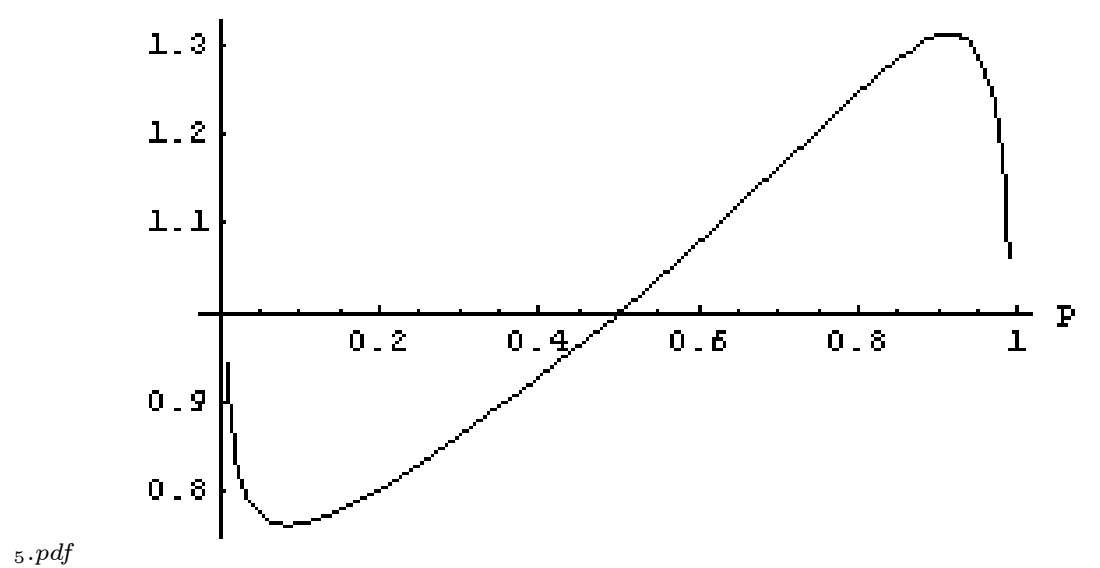

Figure 5: Loss Aversion sufficiency condition for positive risk premium as a function of the probability of a gain $(p)$ with the inverse-S shaped decision weighting function $(\varphi=0.74)$. Restricted range.

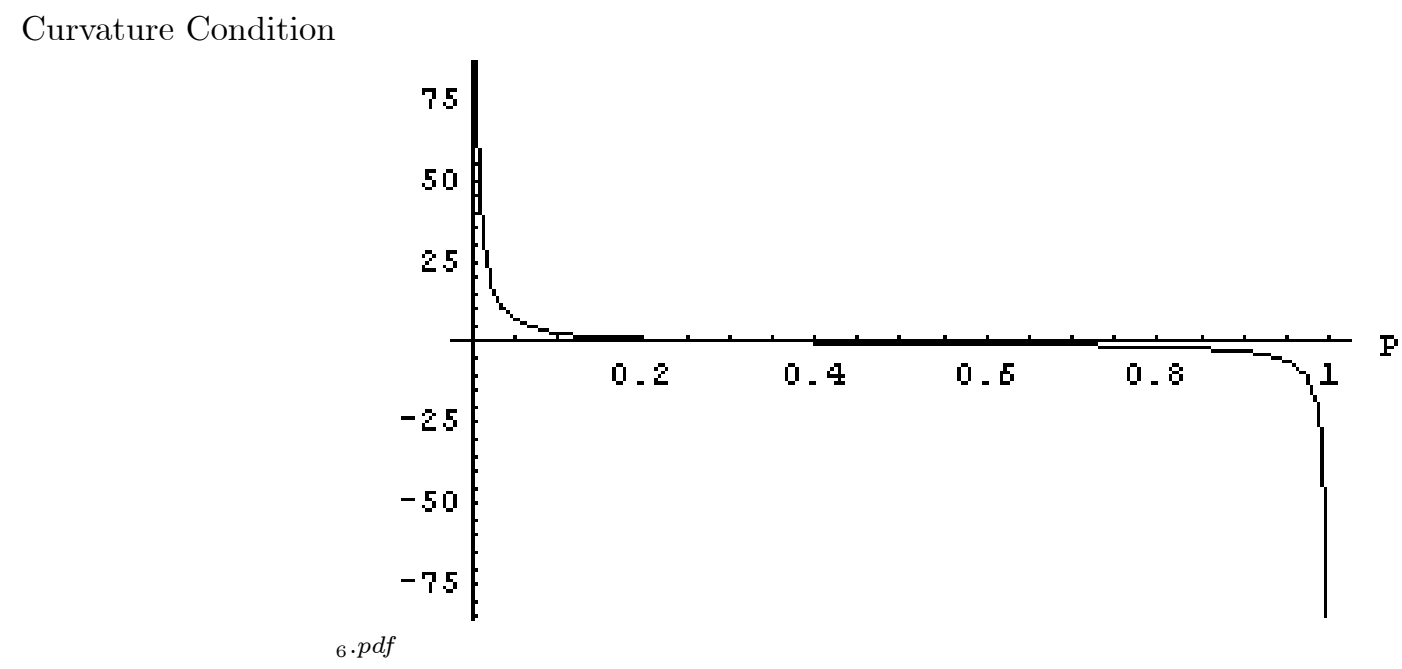

Figure 6: The negative of the relative slope of the muliplier function at the reference point approached from the negative side $\left(-\frac{m_{-}^{\prime}(0)}{m_{-}(0)}\right)$ versus the probability of a gain, $p$. 
Curvature Int Condition

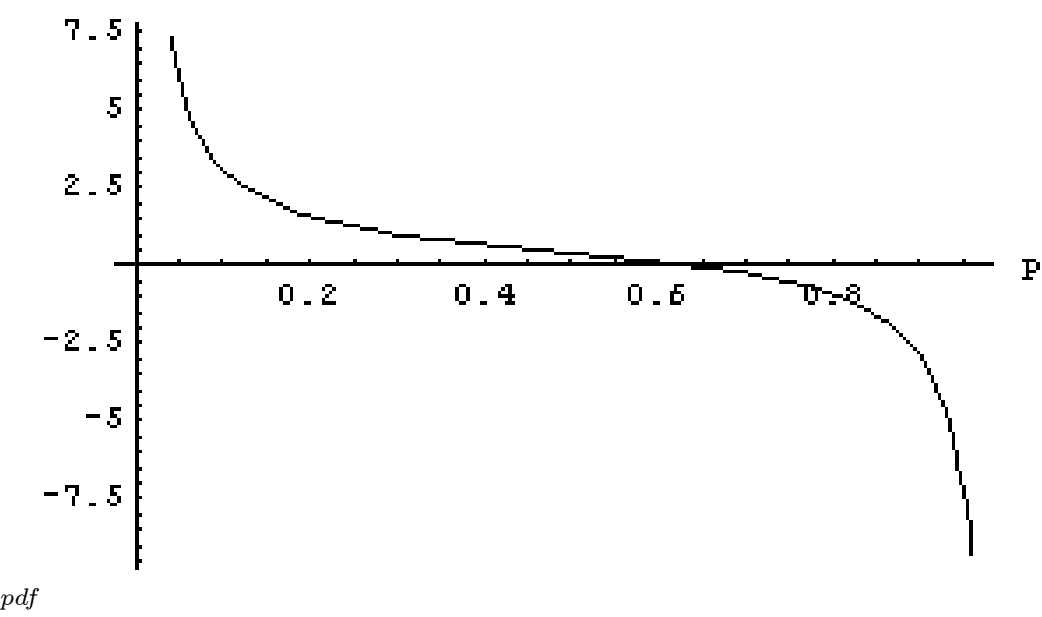

Figure 7: The negative of the relative slope of the muliplier function at the reference point approached from the negative side $\left(-\frac{m_{-}^{\prime}(0)}{m_{-}(0)}\right)$ versus the probability of a gain, $p$. Restricted range.

Curvature Int Condition

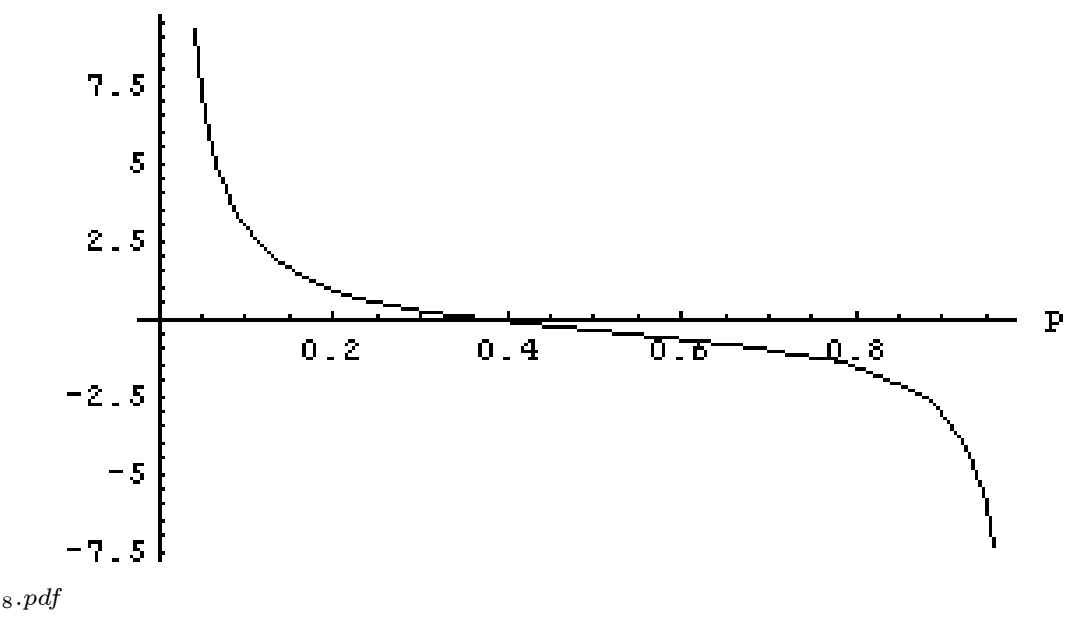

Figure 8: The negative of the relative slope of the muliplier function at the reference point approached from the postive side $\left(-\frac{m_{+}^{\prime}(0)}{m_{+}(0)}\right)$ versus the probability of a gain, $p$. 


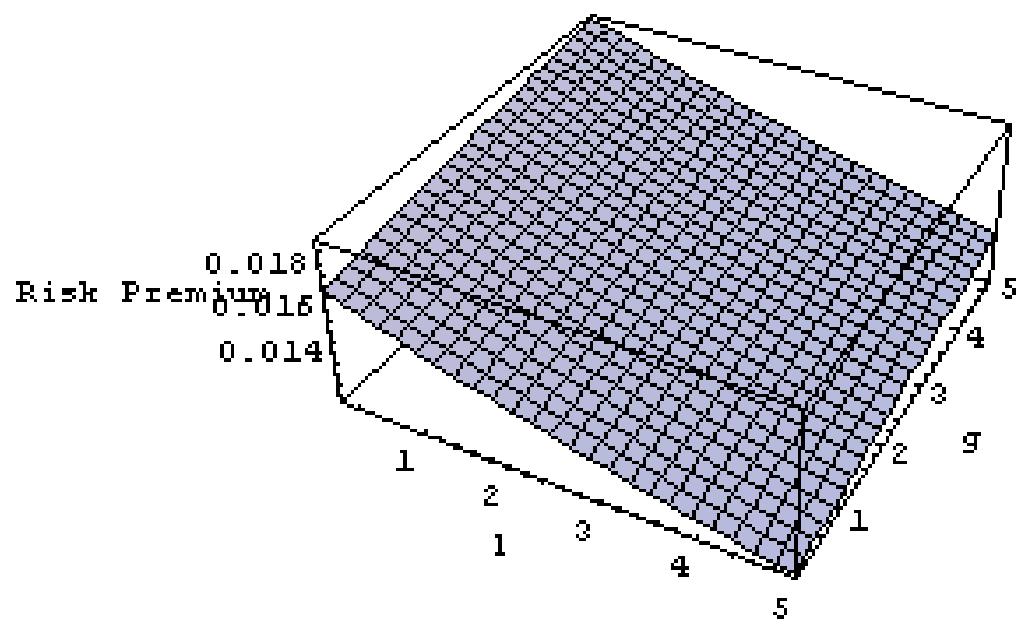

Figure 9: Objective risk premium, $r^{\Psi}$, for concave gains utility $(0<g<5)$ and convex loss utility $(0<l<5)$, with Prelec's inverse-S shaped probability weighting curve $(\varphi=0.74)$, and loss aversion $\lambda=2.25$. 


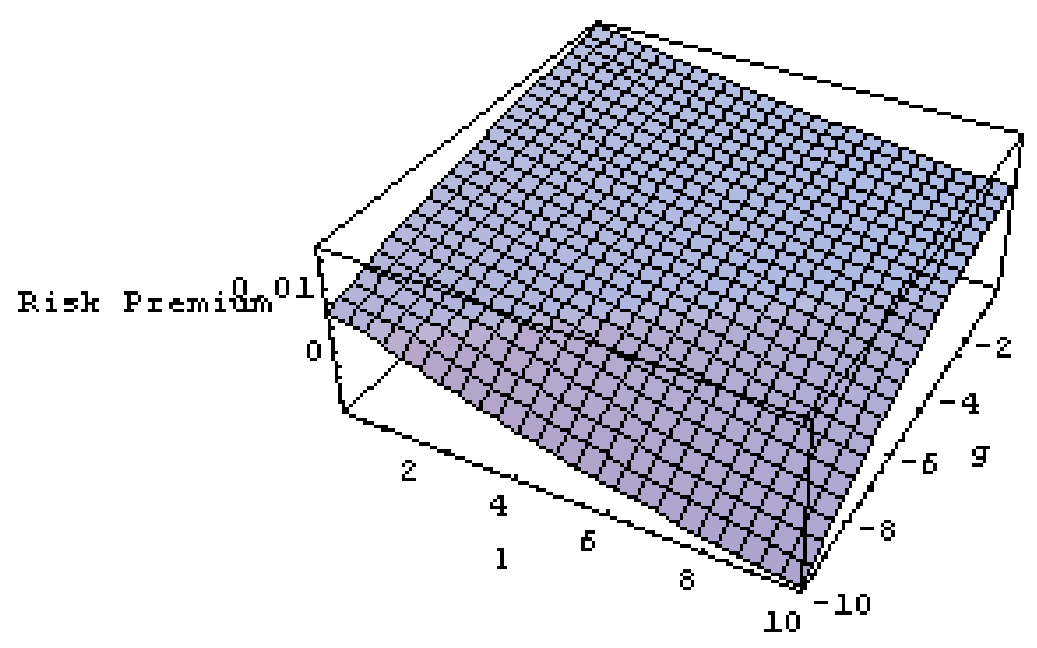

Figure 10: Objective risk premium, $r^{\Psi}$, for convex gains utility $(-10<g<0)$ and convex loss utility $(0<l<10)$, with Prelec's inverse-S shaped probability weighting curve $(\varphi=0.74)$, and loss aversion $\lambda=2.25$. Kink in function occurs at $r^{\Psi}=0.00671$. 
pre

lam and

pre

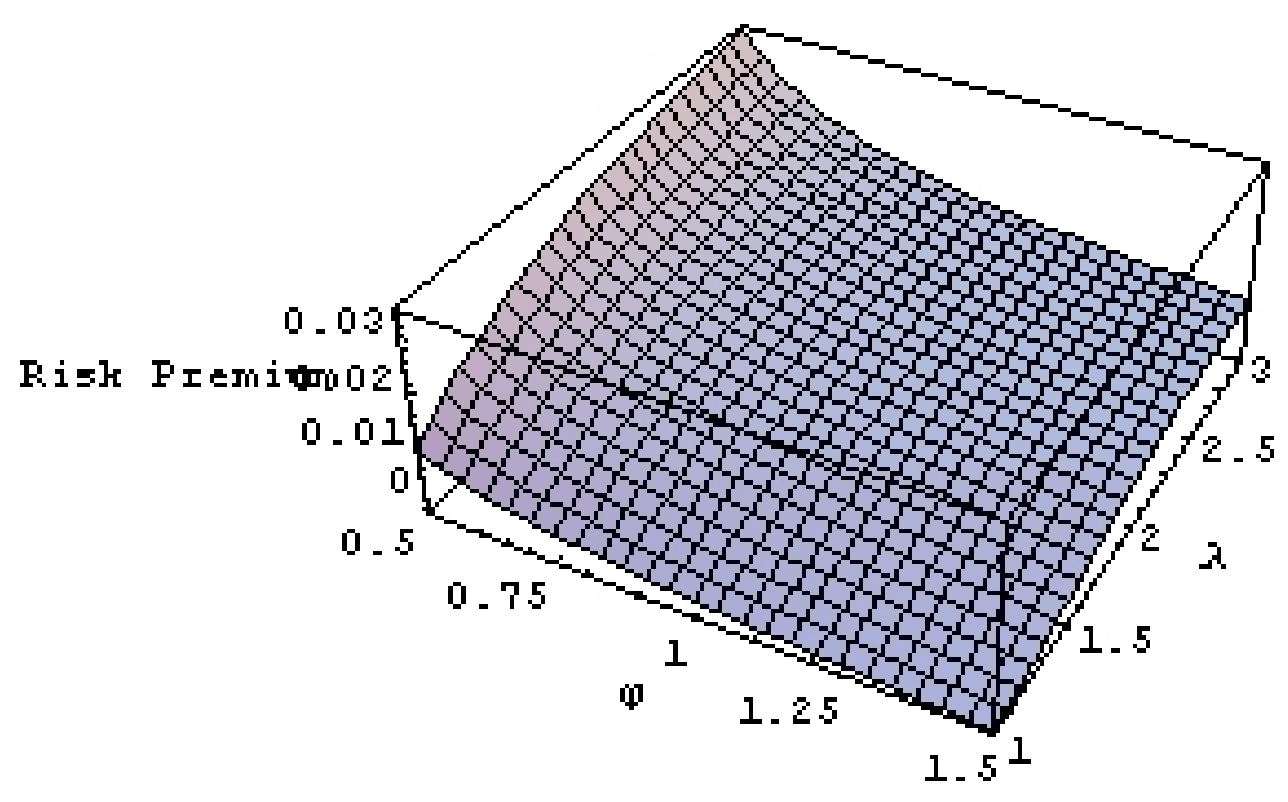

${ }_{1} 1 . p d f$

Figure 11: Objective risk premium, $r^{\Psi}$, for combinations of loss aversion $(\lambda)$ and Prelec's probability weighting curve $(\varphi)$. Gains utility is concave $(g=1)$ and loss utility convex $(l=1)$. Prelec's function is inverse-S shaped for $\varphi<1$, S shaped for $\varphi>1$ with no distortion at $\varphi=1$ Kink in function occurs at $r^{\Psi}=0.00671$. 


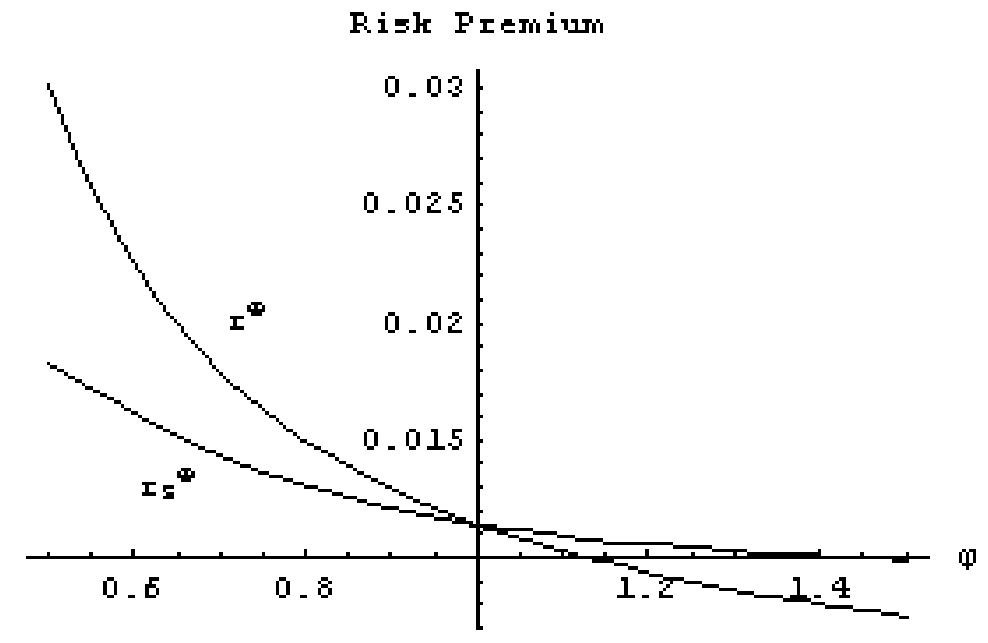

Figure 12: Objective and subjective risk premia for differing degrees of probability distortion $(0.5<\varphi<1.5) . g=l=1$ and $\lambda=2.25$. The Subjectivity premium $r_{\pi}^{\Psi}=r^{\Psi}-r_{S}^{\Psi}$ is equal to the difference between the objective and subjective expected excess returns $E_{y}[\Psi]-E_{y}^{S}[\Psi]$. 